\title{
MULTIPLE TIME SCALE DYNAMICS IN COUPLED GINZBURG-LANDAU EQUATIONS*
}

\author{
FANGHUA LIN ${ }^{\dagger}$ AND TAI-CHIA LIN ${ }^{\ddagger}$
}

\begin{abstract}
Using a rather simple model of coupled, time-dependent Ginzburg-Landau equations with two order parameters, we demonstrate that the total Hamiltonian energy of the system contains at least three levels describing point vortices, domain walls and configurations. The global in time dynamics contain then also at least three different time scales for nontrivial motions of domain walls, boundaries of domain walls (fractional degree vortices) and paired vortices. In particular, we rigorously show, after an initial time period of adjusting, the domain walls start to move according the motion by the mean-curvature that straighten out the domain walls while the boundaries of such domain walls are essentially fixed. After this motion is completed, the fractional degree vortices begin to move at the next time scale. The motion is relatively simple as it is of constant speed and toward each other to form vortex pairs. Finally, these vortex pairs may move in the final time scale very much like the ordinary vortices in a single time-dependent Ginzburg-Landau equation.
\end{abstract}

\section{Introduction}

In this paper, we study a simple model problem for the time-dependent, coupled Ginzburg-Landau equations with two complex order-parameters. More precisely, we consider

$$
\left\{\begin{array}{l}
\lambda_{\epsilon, \xi}^{-1} u_{t}=\Delta u+\frac{1}{\epsilon^{2}}\left(1-|u|^{2}\right) u-\xi(u \cdot v) v, \\
\lambda_{\epsilon, \xi}^{-1} v_{t}=\Delta v+\frac{1}{\epsilon^{2}}\left(1-|v|^{2}\right) v-\xi(u \cdot v) u
\end{array}\right.
$$

with the Neumann boundary conditions

$$
\partial_{\nu} u=\partial_{\nu} v=0
$$

on the boundary $\partial \Omega$, where $\Omega$ is a bounded smooth domain in $\mathbb{R}^{2}$, and $u$ and $v$ are complex-valued functions. Hereafter, $(a \cdot b)$ is defined as $\frac{1}{2}\left(a^{*} b+a b^{*}\right)$ for all $a, b \in \mathbb{C}$, where the asterisk denotes the complex conjugate. We shall always assume that parameters $\epsilon$ and $\xi$ satisfy:

$$
1<<\xi<<\log \frac{1}{\epsilon}
$$

Also $\lambda_{\epsilon, \xi}>0$ is a proper scaling parameter which will be chosen in various cases.

The system (1.1) can be written as

$$
\lambda_{\epsilon, \xi}^{-1} \frac{\partial}{\partial t}(u, v)=-\frac{\delta E_{\epsilon, \xi}(u, v)}{\delta(u, v)},
$$

that is, a gradient flow of the energy functional $E_{\epsilon, \xi}$ defined by

$$
\begin{aligned}
E_{\epsilon, \xi}(u, v)= & \int_{\Omega} \frac{1}{2}\left[|\nabla u|^{2}+|\nabla v|^{2}\right] \\
& +\int_{\Omega} \frac{1}{4 \epsilon^{2}}\left[\left(1-|u|^{2}\right)^{2}+\left(1-|v|^{2}\right)^{2}\right] \\
& +\int_{\Omega} \frac{1}{2} \xi(u \cdot v)^{2} .
\end{aligned}
$$

*Received: September 25, 2003; accepted (in revised version): September 30, 2003. Communicated by Jack Xin.

${ }^{\dagger}$ Courant Institute of Mathematical Sciences, New York, USA.

$\ddagger$ Department of Mathematics, Chung-Cheng Univ., Chia-Yi, Taiwan. 
Our study of (1.1) with the Hamiltonian energy given by (1.4) is motivated by physics literature (cf. [5], [22]) for two superconducting phases in zero field that may contain many interesting new effects and phenomena. In the absence of magnetic fields, the free energy for such superconductors takes the form

$$
\begin{aligned}
F\left(\eta_{1}, \eta_{2}\right)=\int_{\Omega} & K_{1}\left(\left|\partial_{x} \eta_{1}\right|^{2}+\left|\partial_{y} \eta_{2}\right|^{2}\right)+K_{2}\left(\left|\partial_{x} \eta_{2}\right|^{2}+\left|\partial_{y} \eta_{1}\right|^{2}\right)+f_{\text {pot }}\left(\eta_{1}, \eta_{2}\right) \\
& +K_{3}\left(\partial_{x} \eta_{1}^{*} \partial_{y} \eta_{2}+\text { c.c. }\right)+K_{4}\left(\partial_{x} \eta_{2}^{*} \partial_{y} \eta_{1}+\text { c.c. }\right) d x d y
\end{aligned}
$$

for $\eta_{1}$ and $\eta_{2}$ are complex-valued order parameters, where $K_{j}, j=1, \cdots, 4$ are material constants and the asterisk denotes the complex conjugate. Besides,

$$
\begin{aligned}
f_{\text {pot }}\left(\eta_{1}, \eta_{2}\right)= & -\alpha_{0}\left(\left|\eta_{1}\right|^{2}+\left|\eta_{2}\right|^{2}\right)+\alpha_{1}\left(\left|\eta_{1}\right|^{2}+\left|\eta_{2}\right|^{2}\right)^{2} \\
& +\alpha_{2}\left(\eta_{1}^{*} \eta_{2}-\eta_{1} \eta_{2}^{*}\right)^{2}+\alpha_{3}\left|\eta_{1}\right|^{2}\left|\eta_{2}\right|^{2}
\end{aligned}
$$

where $\alpha_{j}$ 's are constants. In a simple situation with two component order parameters $\eta_{i}, i=1,2$, the expression (1.5) from traditional Ginzburg-Landau theory agrees quite well with that constructed from an another group-theoretic argument (cf. [19] pp.277).

To capture some new interesting phenomena described by (1.5) and its associated flow, we assume in this paper that $K_{1}=K_{2}=K=\frac{1}{2}, K_{3}=K_{4}=0, \alpha_{0}=$ $\frac{1}{2 \epsilon^{2}}, \alpha_{1}=\frac{1}{4 \epsilon^{2}}, \alpha_{2}=\frac{1}{8} \xi, \alpha_{3}=4 \alpha_{2}-2 \alpha_{1}$. Under these particular choices of material constants, we lead to (1.1). Even with such specific choices of material constants, we would like to point out that our choices of parameters remain to verify $\alpha_{2}>0$, $\alpha_{3}<4 \alpha_{2}, 4\left(\alpha_{1}-\alpha_{2}\right)+\alpha_{3}>0$. It is apparently important that these parameters stay in the region so-called time-reversal symmetry breaking state which leads to many phenomena unconventional to traditional superconductors. In conventional superconductors, the phase winding around a vortex center is an integer multiple of $2 \pi$, and the winding numbers of vortices are integers. However, vortices with a fractional winding number $\frac{1}{2}$ have been predicted to occur in heavy-fermion superconductors (cf. [6]) and have recently been identified in high- $T_{c}$ superconducting rings on tricrystal substrate geometries (cf. [9]). It is known to physicists, vortices for unconventional superconductors generically have a non-universal flux quantum due to the structure of domain walls. Such a structure often makes vortices become fractional degree vortices, and the winding number of each vortex is a multiple of a half, see e.g. [18] and [[19], pp. 285-287].

From [17], domain walls occupied with strongly pinned fractional degree vortices, represent efficient barriers for vortex motion and thus prevent relaxation towards equilibrium. However, the interaction of the motion of domain walls and the dynamical law of fractional degree vortices is still unclear. The main purpose of this paper is to present rigorous proofs (even though they could be at a rather simple and specific situation) how such domain walls and fractional degree vortices can be formed, and how they evolve in time according to the system (1.1) at the asymptotic limits when parameters $1<<\xi<<\log \frac{1}{\epsilon}$. We derive three time scales for the motion of domain walls and fractional degree vortices. In the first time scale of order $O(\sqrt{\xi})$, curved domain walls do a motion by the mean curvature while fractional degree vortices located at the boundaries of domain walls are essentially static. This is consistent with the experimental observation of [4]. Then in the second time scale of order $O\left(\left(\log \frac{1}{\epsilon}\right) / \sqrt{\xi}\right)$, domain walls have become line segments and fractional degree vortices start to move toward each other in pairs. Eventually, in the third time scale of order $O\left(\log \frac{1}{\epsilon}\right)$, fractional degree vortices have recombined in pairs and behave like 
conventional vortices. Such a new dynamical phenomenon is different from recombining fractional degree vortices which is forced by growing density of vortices close to the domain wall (cf. [17] Section 3.2).

The paper is described as follows. In section 2 , we consider minimum energy configurations (naturally we should consider the Dirichlet boundary conditions for order parameters in this case) with the coupling constant $\xi$ fixed while $\epsilon \rightarrow 0^{+}$. This can be viewed as a relatively weak coupling situation. We establish the minimal energy asymptotics in terms of scalar parameters $\epsilon$ and $\xi$. Here we see the minimal energy has three levels (so long as $\xi$ is suitably large) : $O(1), O(\sqrt{\xi})$ and $O\left(\log \frac{1}{\epsilon}\right)$. This fact is responsible for the multiple time scales involved in the global dynamics of (1.1). In order to understand sharp domain walls and fractional degree vortices (which one should be able to see approximately when $\xi$ is suitably large) on the boundaries of such domain walls, we consider another asymptotic in section 3 . If we first let $\epsilon \rightarrow 0^{+}$while keeping $\xi$ fixed, and then let $\xi \rightarrow+\infty$, we can examine the behavior of domain walls as well as degree $\frac{1}{2}$ vortices at the boundaries of these domain walls. If the boundaries of domain walls are fixed, we show the domain walls have to become straight line segments. Various simple analyses are also carried out for the case $1<<\xi<<\log \frac{1}{\epsilon}$, and $\xi \rightarrow \infty$. In general, the limiting configuration can not have domain walls except those due directly to the boundary conditions, and all vortices form pairs (those extra ones presumably go to the boundary of the physical sample). Section 4 is denoted to study dynamics. Vortex dynamics is now well understood for conventional superconductors described by a single Ginzburg-Landau equation (see [11], [12]). Generally speaking, vortices are essentially static in $O(1)$ time scale but vortices have a well-defined motion law (given by an explicitly O.D.E. system) in the time scale of $O\left(\log \frac{1}{\epsilon}\right)$. In other words, it takes exactly $O\left(\log \frac{1}{\epsilon}\right)$ time to move a vortex by an appreciable distance $O(1)$. For unconventional superconductors, the situation is much more complex. For the system (1.1) with suitably chosen initial data (or after an initial $O(1)$ time period of adjusting data), when the parameters $\xi$ and $\epsilon$ satisfy $1<<\xi<<\log \frac{1}{\epsilon}$, one first sees curved domain walls do a motion by the mean curvature while the boundaries of domain walls are essentially static in the time scale of order $O(\sqrt{\xi})$. After this dynamic is over, all domain walls become straight (line segments) with boundaries of domain walls being those degree $\frac{1}{2}$ vortices. Then in the next time scale of order $O\left(\left(\log \frac{1}{\epsilon}\right) / \sqrt{\xi}\right)$ which by our assumption is much larger than $O(\sqrt{\xi})$, these fractional degree vortices at the boundary of straight domain walls move at a constant speed toward each other to reduce the length of domain walls, and to form vortex pairs. Finally, if these vortex pairs are not at the optimal positions (critical to a function involving only the locations of vortices) at the end of time scale $O\left(\left(\log \frac{1}{\epsilon}\right) / \sqrt{\xi}\right)$, then these pairs would undergo a simple motion governed by an explicit O.D.E. in the time scale $O\left(\log \frac{1}{\epsilon}\right)$ which is very much like a single timedependent Ginzburg-Landau equation.

\section{Minimization of Two-component Order Parameter}

In this section, we let $\epsilon \rightarrow 0+$ and fix $\xi$ as any positive constant, and we consider minimization of the energy functional $E_{\epsilon, \xi}$ defined in (1.4) for $u, v \in H^{1}(\Omega ; \mathbb{C})$ with Dirichlet boundary conditions

$$
u=g_{1}, \quad v=g_{2} \text { on } \partial \Omega,
$$

where $g_{j}: \partial \Omega \rightarrow S^{1}$ are smooth maps with degree $d_{j} \in \mathbf{N}$ for $j=1,2$. Now we state our main result on such a minimizing problem as follows: 
Theorem I. Fix $\xi$ as any positive constant. Let $\left(v_{1, \epsilon}, v_{2, \epsilon}\right)$ be the minimizer of the energy functional $E_{\epsilon, \xi}$ defined in (1.4) on the function space $\left\{\left(v_{1}, v_{2}\right): v_{j} \in H_{g_{j}}^{1}(\Omega ; \mathbb{C})\right.$, $j=1,2\}$, where $g_{j}: \partial \Omega \rightarrow S^{1}$ is a smooth map with degree $d_{j} \in \mathbf{N}$ for $j=1,2$. Then

(i) $\quad v_{j, \epsilon}$ has $d_{j}$ degree-one vortices in $\Omega, j=1,2$.

(ii) $v_{j, \epsilon}$ converges to $v_{j}^{*}$ (up to a subsequence) strongly in $L^{2}(\Omega)$ and weakly in $H_{\text {loc }}^{1}\left(\Omega \backslash\left\{a_{1}^{j}, \cdots, a_{d_{j}}^{j}\right\}\right)$, where $v_{j}^{*}$ is defined by

$$
v_{j}^{*}(z)=\prod_{k=1}^{d_{j}} \frac{z-a_{k}^{j}}{\left|z-a_{k}^{j}\right|} e^{i h_{a^{j}}(z)}, \quad \forall z \in \Omega,
$$

and $h_{a^{j}}$ 's satisfy

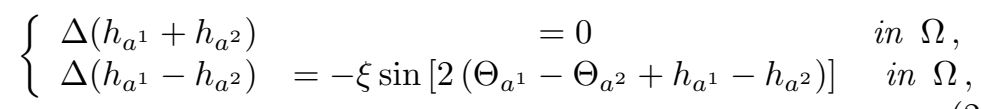

where $\Theta_{a^{j}}=\sum_{k=1}^{d_{j}} \arg \left(\frac{z-a_{k}^{j}}{\left|z-a_{k}^{j}\right|}\right)$, and $a^{j}=\left(a_{1}^{j}, \cdots, a_{d_{j}}^{j}\right) \in \Omega^{d_{j}}$.

(iii) $\tilde{E}_{\epsilon}\left(v_{1, \epsilon}, v_{2, \epsilon}\right)=\pi \sum_{j=1}^{2} d_{j} \log \frac{1}{\epsilon}+W_{g_{1}, g_{2}}\left(a^{1}, a^{2}\right)+\sum_{j=1}^{2} d_{j} \gamma+o_{\epsilon}(1)$ as $\epsilon \rightarrow 0+$, where $\gamma>0$ is a universal constant. Here $W_{g_{1}, g_{2}}$ is the renormalized energy defined by

$W_{g_{1}, g_{2}}\left(a^{1}, a^{2}\right)=\lim _{\rho \rightarrow 0+} \int_{\Omega_{\rho}} \sum_{j=1}^{2} \frac{1}{2}\left|\nabla w_{j}\right|^{2}+\frac{1}{2} \xi\left(w_{1} \cdot w_{2}\right)^{2}-\pi \sum_{j=1}^{2} d_{j} \log \frac{1}{\rho}$,

where $\Omega_{\rho}=\Omega \backslash \cup_{j=1}^{2} \cup_{k=1}^{d_{j}} B_{\rho}\left(a_{k}^{j}\right),\left(w_{1}, w_{2}\right)$ is the energy minimizer of the energy functional

$$
\int_{\Omega_{\rho}} \sum_{j=1}^{2} \frac{1}{2}\left|\nabla v_{j}\right|^{2}+\frac{1}{2} \xi\left(v_{1} \cdot v_{2}\right)^{2}
$$

for $v_{j} \in H_{l o c}^{1}\left(\Omega \backslash\left\{a_{1}^{j}, \cdots, a_{d_{j}}^{j}\right\} ; S^{1}\right)$ satisfying

$\left.v_{j}\right|_{\partial \Omega}=g_{j}$ and $\operatorname{deg}\left(v_{j} ; \partial B_{\rho_{0}}\left(a_{k}^{j}\right)\right)=1 \quad$ for $j=1,2, k=1, \cdots, d_{j}$,

where $\rho_{0}$ is a positive constant.

(iv) $\quad\left(a^{1}, a^{2}\right) \in \Omega^{d_{1}} \times \Omega^{d_{2}}$ is a global minimizer of $W_{g_{1}, g_{2}}\left(b^{1}, b^{2}\right)$ for $b^{j}=\left(b_{1}^{j}, \cdots, b_{d_{j}}^{j}\right) \in \Omega^{d_{j}}, j=1,2$.

As $\xi=0,(2.3)$ implies that $h_{a^{j}}$ 's are harmonic functions and can be uniquely determined by the boundary conditions. Moreover, $W_{g_{1}, g_{2}}\left(a^{1}, a^{2}\right)=\sum_{j=1}^{2} W_{g_{j}}\left(a^{j}\right)$. However, if $\xi>0,(2.3)$ can be written as

$$
\begin{aligned}
& \Delta \Phi_{1}=0 \quad \text { in } \Omega, \\
& \Delta \Phi_{2}=-\xi \sin \left[2\left(\Theta_{a^{1}}-\Theta_{a^{2}}+\Phi_{2}\right)\right] \text { in } \Omega,
\end{aligned}
$$


where $\Phi_{1}=h_{a^{1}}+h_{a^{2}}$ and $\Phi_{2}=h_{a^{1}}-h_{a^{2}}$. It is remarkable that (2.4) is a Poisson equation and (2.5) is an elliptic sine-Gordon equation. As $\xi$ goes to infinity, the standard theorem of singular perturbation problems implies that the equation (2.5) may have a solution with an interface in $\Omega$. Such an interface is called a domain wall(cf. [20]). In Section 3, we will study the structure of a domain wall.

To prove Theorem I, we need three propositions as follows:

Proposition I. Let $\tilde{v}_{\epsilon}$ be the energy minimizer of the energy functional $\int_{\Omega} e_{\epsilon}(v)$ on $H_{g}^{1}(\Omega ; \mathbb{C})$, where $g: \partial \Omega \rightarrow S^{1}$ is a smooth map with degree $d \in \mathbf{N}$, and

$$
e_{\epsilon}(v) \equiv \frac{1}{2}|\nabla v|^{2}+\frac{1}{4 \epsilon^{2}}\left(1-|v|^{2}\right)^{2} .
$$

Then

(i) $\tilde{v}_{\epsilon}$ has $d$ degree-one vortices in $\Omega$,

(ii) $\int_{\Omega} e_{\epsilon}\left(\tilde{v}_{\epsilon}\right)=\pi d \log \frac{1}{\epsilon}+O(1)$ as $\epsilon \rightarrow 0+$.

(iii) $\tilde{v}_{\epsilon}$ converges to $v_{*}$ (up to a subsequence) in $C_{l o c}^{1, \alpha}\left(\Omega \backslash\left\{a_{1}, \cdots, a_{d}\right\}\right)$, for $0<\alpha<1$, where $v_{*}$ is a canonical harmonic map defined by

$$
v_{*}(z)=\prod_{j=1}^{d} \frac{z-a_{j}}{\left|z-a_{j}\right|} e^{i h_{a}(z)}, \quad \forall z \in \Omega,
$$

and $h_{a}$ is a real-valued harmonic function.

Proposition II. Suppose $v_{\epsilon} \in H_{g}^{1}(\Omega ; \mathbb{C})$ such that

$$
\int_{\Omega} e_{\epsilon}\left(v_{\epsilon}\right) \leq \pi d \log \frac{1}{\epsilon}+C_{0} \quad \text { as } \epsilon \rightarrow 0+
$$

where $C_{0}$ is a positive constant independent of $\epsilon$. Then, by taking a subsequence if necessary, we have as $\epsilon \rightarrow 0+$ that:

$$
\frac{e_{\epsilon}\left(v_{\epsilon}\right) d x d y}{\pi d \log \frac{1}{\epsilon}} \rightarrow \sum_{j=1}^{d} \delta_{a_{j}},
$$

as Radon measures. Here $a_{j}, j=1, \cdots, d$ are $d$ distinct points inside $\Omega$ such that

$$
\min \left\{\left|a_{i}-a_{j}\right|, \operatorname{dist}\left(a_{i}, \partial \Omega\right): i, j=1, \cdots, d, i \neq j\right\} \geq \delta_{0}\left(z, \Omega, C_{0}\right)>0,
$$

and $v_{\epsilon}$ converges (up to a subsequence) to a map of the form

$$
\prod_{j=1}^{d} \frac{z-a_{j}}{\left|z-a_{j}\right|} e^{i h_{a}(z)}
$$

strongly in $L^{2}(\Omega)$ and weakly in $H_{l o c}^{1}\left(\bar{\Omega} \backslash\left\{a_{1}, \cdots, a_{d}\right\}\right)$ as $\epsilon \rightarrow 0+$. Moreover,

$$
\left\|h_{a}\right\|_{H^{1}(\Omega)} \leq C\left(C_{0}, z, \Omega\right) .
$$


Proposition III. Under the same assumptions of Proposition II, the linear momentum $p\left(v_{\epsilon}\right) \equiv i v_{\epsilon} \cdot \nabla v_{\epsilon}$ is uniformly bounded in $L_{\text {loc }}^{1}\left(\Omega_{a}\right)$, and up to a subsequence if necessary:

$$
p\left(v_{\epsilon}\right) \rightarrow \nabla \Theta_{a}+\nabla h_{a}
$$

in $L_{l o c}^{1}\left(\Omega_{a}\right)$, where $\Omega_{a} \equiv \Omega \backslash\left\{a_{1}, \cdots, a_{d}\right\}$ and $\Theta_{a}=\sum_{j=1}^{d} \arg \left(\frac{z-a_{j}}{\left|z-a_{j}\right|}\right)$. Moreover,

$$
2 J_{a c}\left(v_{\epsilon}\right) d x d y=\operatorname{curl}\left(p\left(v_{\epsilon}\right)\right) d x d y \rightarrow 0,
$$

in the sense of bounded measures $M\left(\Omega_{a}\right)$, where $J_{a c}\left(v_{\epsilon}\right)$ is the Jacobian of $v_{\epsilon}$.

One may find the proof of Proposition I in [3] and [11]. Proposition II and III were proved in [13] and [14], respectively. Now we prove Theorem I as follows:

Let $\left(v_{1, \epsilon}, v_{2, \epsilon}\right)$ be the minimizer of the energy functional $E_{\epsilon, \xi}$ defined in (1.4) on the function space $\left\{\left(v_{1}, v_{2}\right): v_{j} \in H_{g_{j}}^{1}(\Omega ; \mathbb{C}), j=1,2\right\}$, where $g_{j}: \partial \Omega \rightarrow S^{1}$ is a smooth map with degree $d_{j} \in \mathbf{N}$. From the standard Direct method, it is easy to obtain the existence of the energy minimizer $\left(v_{1, \epsilon}, v_{2, \epsilon}\right)$. Let $w_{j, \epsilon}$ be the energy minimizer of the energy functional $\tilde{E}_{\epsilon}(w) \equiv \int_{\Omega} \frac{1}{2}|\nabla w|^{2}+\frac{1}{4 \epsilon^{2}}\left(1-|w|^{2}\right)^{2}$ on $H_{g_{j}}^{1}(\Omega ; \mathbb{C})$. From [3], the quantitative properties of $w_{j, \epsilon}$ 's are well-known. It is obvious that

$$
\tilde{E}_{\epsilon}\left(w_{j, \epsilon}\right)=\pi d_{j} \log \frac{1}{\epsilon}+O(1), \quad j=1,2,
$$

where $O(1)$ is a bounded quantity independent of $\epsilon$. By energy comparison, we have

$$
\int_{\Omega} e_{\epsilon}\left(v_{j, \epsilon}\right)=\pi d_{j} \log \frac{1}{\epsilon}+O(1)
$$

where $e_{\epsilon}(w) \equiv \frac{1}{2}|\nabla w|^{2}+\frac{1}{4 \epsilon^{2}}\left(1-|w|^{2}\right)^{2}$. Then by Proposition II, III and (2.14), we may complete the proof of Theorem I except (2.3). To prove (2.3), we consider the Euler-Lagrange equations of $v_{j, \epsilon}, j=1,2$ given by

$$
\begin{aligned}
& \Delta v_{1, \epsilon}+\frac{1}{\epsilon^{2}}\left(1-\left|v_{1, \epsilon}\right|^{2}\right) v_{1, \epsilon}=\xi\left(v_{1, \epsilon} \cdot v_{2, \epsilon}\right) v_{2, \epsilon} \quad \text { in } \Omega, \\
& \Delta v_{2, \epsilon}+\frac{1}{\epsilon^{2}}\left(1-\left|v_{2, \epsilon}\right|^{2}\right) v_{2, \epsilon}=\xi\left(v_{1, \epsilon} \cdot v_{2, \epsilon}\right) v_{1, \epsilon} \quad \text { in } \Omega .
\end{aligned}
$$

From (2.15) and (2.16), we have

$$
\int_{\Omega}\left(\Delta v_{1, \epsilon} \cdot i v_{1, \epsilon}\right) \psi=\xi \int_{\Omega}\left(v_{1, \epsilon} \cdot v_{2, \epsilon}\right)\left(v_{2, \epsilon} \cdot i v_{1, \epsilon}\right) \psi,
$$

and

$$
\int_{\Omega}\left(\Delta v_{2, \epsilon} \cdot i v_{2, \epsilon}\right) \psi=\xi \int_{\Omega}\left(v_{1, \epsilon} \cdot v_{2, \epsilon}\right)\left(v_{1, \epsilon} \cdot i v_{2, \epsilon}\right) \psi,
$$

for any test function $\psi \in C_{0}^{\infty}(\Omega ; \mathbb{R})$. Using integration by parts and Proposition II, 
we may obtain

$$
\begin{aligned}
& \int_{\Omega} p\left(v_{1, \epsilon}\right) \nabla \psi=-\int_{\Omega}\left(\Delta v_{1, \epsilon} \cdot i v_{1, \epsilon}\right) \psi \quad \text { (integration by parts) } \\
&=-\xi \int_{\Omega}\left(v_{1, \epsilon} \cdot v_{2, \epsilon}\right)\left(v_{2, \epsilon} \cdot i v_{1, \epsilon}\right) \psi \quad(\text { by }(2.17)) \\
&=-\xi \int_{\Omega}\left[e^{i\left(\Theta_{a^{1}}+h_{a^{1}}\right)} \cdot e^{i\left(\Theta_{a^{2}}+h_{a^{2}}\right)}\right] \\
& \times\left[e^{i\left(\Theta_{a^{2}}+h_{a^{2}}\right)} \cdot i e^{i\left(\Theta_{a^{1}}+h_{a^{1}}\right)}\right] \psi d x+o_{\epsilon}(1) \quad(\text { by }(2.2)),
\end{aligned}
$$

i.e.

$$
\begin{aligned}
\int_{\Omega} p\left(v_{1, \epsilon}\right) \nabla \psi=-\xi \int_{\Omega} & {\left[e^{i\left(\Theta_{a^{1}}+h_{a^{1}}\right)} \cdot e^{i\left(\Theta_{a^{2}}+h_{a^{2}}\right)}\right] } \\
\times & \left.\times e^{i\left(\Theta_{a^{2}}+h_{a^{2}}\right)} \cdot i e^{i\left(\Theta_{a^{1}}+h_{a^{1}}\right)}\right] \psi d x+o_{\epsilon}(1),
\end{aligned}
$$

for any test function $\psi \in C_{0}^{\infty}(\Omega ; \mathbb{R})$. Similarly, we have

$$
\begin{aligned}
\int_{\Omega} p\left(v_{2, \epsilon}\right) \nabla \psi=-\xi \int_{\Omega} & {\left[e^{i\left(\Theta_{a^{1}}+h_{a^{1}}\right)} \cdot e^{i\left(\Theta_{a^{2}}+h_{a^{2}}\right)}\right] } \\
& \times\left[e^{i\left(\Theta_{a^{1}}+h_{a^{1}}\right)} \cdot i e^{i\left(\Theta_{a^{2}}+h_{a^{2}}\right)}\right] \psi d x+o_{\epsilon}(1),
\end{aligned}
$$

for any test function $\psi \in C_{0}^{\infty}(\Omega ; \mathbf{R})$. Therefore by (2.19), (2.20) and Proposition III, we may obtain (2.3) and we complete the proof of Theorem I.

\section{Domain Wall of Phase Functions}

To see the effect of strong coupling of phase functions, we study the minimization problem of the energy functional (1.4) given by

$$
\begin{aligned}
E_{\epsilon, \xi}(u, v)= & \int_{\Omega} \frac{1}{2}|\nabla u|^{2}+\frac{1}{4 \epsilon^{2}}\left(1-|u|^{2}\right)^{2} d x d y \\
& +\int_{\Omega} \frac{1}{2}|\nabla v|^{2}+\frac{1}{4 \epsilon^{2}}\left(1-|v|^{2}\right)^{2} d x d y+\frac{1}{2} \xi \int_{\Omega}(u \cdot v)^{2} d x d y
\end{aligned}
$$

for $u$ and $v \in H^{1}(\Omega ; \mathbb{C})$ with Dirichlet boundary conditions having the same degree $d$. Hereafter, we assume that $\xi \gg 1$ is a large constant independent of $\epsilon$. Then the phase functions of $u$ and $v$ have been strongly coupled by the term $\frac{1}{2} \xi \int_{\Omega}(u \cdot v)^{2}$. As $\epsilon$ goes to zero and fix $\xi$ as any constant, Theorem I implies the energy minimizer $\left(u_{\epsilon}, v_{\epsilon}\right)$ satisfying that up to a subsequence, $u_{\epsilon}$ converges to $u_{*}$ and $v_{\epsilon}$ converges to $v_{*}$ strongly in $L^{2}(\Omega)$ and weakly in $H_{l o c}^{1}\left(\Omega \backslash\left\{a_{1}, \cdots, a_{d}\right\}\right)$ and $H_{l o c}^{1}\left(\Omega \backslash\left\{b_{1}, \cdots, b_{d}\right\}\right)$, respectively. Here $u_{*}$ and $v_{*}$ are defined by

$$
\begin{aligned}
& u_{*}(z)=\prod_{k=1}^{d} \frac{z-a_{k}}{\left|z-a_{k}\right|} e^{i \phi(z)}, \quad \forall z \in \Omega, \\
& v_{*}(z)=\prod_{k=1}^{d} \frac{z-b_{k}}{\left|z-b_{k}\right|} e^{i \Phi(z)}, \quad \forall z \in \Omega,
\end{aligned}
$$


where $\phi$ and $\Phi$ satisfy

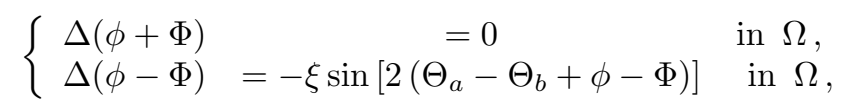

where $\Theta_{a}=\sum_{k=1}^{d} \arg \left(\frac{z-a_{k}}{\left|z-a_{k}\right|}\right), \Theta_{b}=\sum_{k=1}^{d} \arg \left(\frac{z-b_{k}}{\left|z-b_{k}\right|}\right)$, and $a=\left(a_{1}, \cdots, a_{d}\right) \in$ $\Omega^{d}, b=\left(b_{1}, \cdots, b_{d}\right) \in \Omega^{d}$. Then the renormalized energy can be written as

$$
\sqrt{\xi} \int_{\Omega} \frac{1}{4 \sqrt{\xi}}|\nabla H|^{2}+\frac{1}{2} \sqrt{\xi} \cos ^{2}\left(\Theta_{a}-\Theta_{b}+H\right) d x d y+\int_{\Omega} \frac{1}{4}|\nabla h|^{2}+W\left(a, b, \phi_{0}, \Phi_{0}\right),
$$

where $H=\phi-\Phi, h=\phi+\Phi,\left.\phi\right|_{\partial \Omega}=\phi_{0},\left.\Phi\right|_{\partial \Omega}=\Phi_{0}$, and

$$
\begin{aligned}
W\left(a, b, \phi_{0}, \Phi_{0}\right)= & \lim _{\rho \rightarrow 0+} \int_{\Omega_{\rho}} \frac{1}{2}\left|\nabla \Theta_{a}\right|^{2}+\frac{1}{2}\left|\nabla \Theta_{b}\right|^{2}-2 \pi d \log \frac{1}{\rho} \\
& +\int_{\partial \Omega} \phi_{0} \partial_{\vec{n}} \Theta_{a}+\Phi_{0} \partial_{\vec{n}} \Theta_{b}
\end{aligned}
$$

where $\Omega_{\rho}=\Omega \backslash \cup_{k=1}^{d} B_{\rho}\left(a_{k}\right) \cup B_{\rho}\left(b_{k}\right)$, and $\partial_{\vec{n}}$ is the normal derivative on the boundary $\partial \Omega$. Here the boundary conditions $\phi_{0}$ and $\Phi_{0}$ come from the Dirichlet boundary conditions. Now we define the energy density

$$
p_{\xi}(H ; a, b)=\frac{1}{4 \sqrt{\xi}}|\nabla H|^{2}+\frac{1}{2} \sqrt{\xi} \cos ^{2}\left(\Theta_{a}-\Theta_{b}+H\right),
$$

and the energy functional

$$
P_{\xi}(H ; a, b)=\int_{\Omega} p_{\xi}(H ; a, b) d x d y, \quad \text { for } H \in H^{1}(\Omega ; \mathbb{R}), \text { and } a, b \in \Omega^{d} .
$$

As $\xi$ goes to infinity, the leading terms of the renormalized energy $(3.5)$ are $\sqrt{\xi} P_{\xi}(H ; a, b)$. As $a=b$, the energy density

$$
p_{\xi}(H ; a, a)=\frac{1}{4 \sqrt{\xi}}|\nabla H|^{2}+\frac{1}{2} \sqrt{\xi} \cos ^{2}(H),
$$

is independent of $a$. For the minimization of $P_{\xi}$, we have

Proposition IV. Assume $\left(H_{\xi}, a_{\xi}, b_{\xi}\right)$ is the energy minimizer of $P_{\xi}(H ; a, b)$ for $H \in H^{1}(\Omega ; \mathbf{R}), a, b \in \Omega^{d}$. Then $a_{\xi}=b_{\xi}$ and $H_{\xi} \equiv \pi / 2(\bmod \pi)$ is a constant function.

From Proposition IV, we may conclude that the strong coupling of phase functions makes vortices of $u_{\epsilon}$ and $v_{\epsilon}$ in pairs. Vortices in pairs form an interesting phenomenon called vortex confinement which was predicted formally by physicists (cf. [20]). Here we provide a mathematical proof of such a phenomenon. The proof of Proposition IV is obvious. It is easy to check that the constant function $H_{0} \equiv \pi / 2$ is one of the energy minimizers of $P_{\xi}$ on $H^{1}(\Omega ; \mathbb{R})$, and $P_{\xi}\left(H_{0} ; a, a\right)=0$, for all $a \in \Omega$. Then the energy comparison implies that

$$
P_{\xi}\left(H_{\xi} ; a_{\xi}, b_{\xi}\right) \leq P_{\xi}\left(H_{0}, a_{\xi}, a_{\xi}\right)=0
$$

Hence $H_{\xi}$ is a constant function, $a_{\xi}=b_{\xi}$, and we complete the proof of Proposition IV. 
Proposition IV shows that vortices of $u_{\epsilon}$ and $v_{\epsilon}$ would like to come together in pairs if we ignore the effect of the Dirichlet boundary condition $\left.H\right|_{\partial \Omega}=\phi_{0}-$ $\Phi_{0}$. If we consider the Dirichlet boundary condition $\left.H\right|_{\partial \Omega}=\phi_{0}-\Phi_{0}$, then the energy minimizer $H_{\xi}$ may have a boundary layer near the boundary $\partial \Omega$ to adjust the boundary condition.

To see the minimizing energy for vortices not in pairs, we fix $a, b \in \Omega, a \neq b$, and minimize the energy functional $P_{\xi}(\cdot ; a, b)$ on $H^{1}(\Omega ; \mathbf{R})$. Then we have

Theorem II. Assume $\Omega$ is a smooth and bounded domain in $\mathbf{R}^{2}$. Fix two distinct points $a, b \in \Omega$, such that $a$ and $b$ are independent of $\xi$, and the line segment $L$ joining $a$ and $b$ is located in the interior of $\Omega$. Let $H_{\xi}$ be the energy minimizer of $P_{\xi}(H ; a, b)$ for $H \in H^{1}(\Omega ; \mathbb{R})$ with the Dirichlet boundary condition $H=\Theta_{b}-\Theta_{a}+\frac{1}{2} \pi$, or the Neumann boundary condition $\partial_{\nu} H=\partial_{\nu}\left(\Theta_{b}-\Theta_{a}\right)$ on the boundary $\partial \Omega$. Then

$$
\begin{gathered}
H_{\xi} \rightarrow \Theta_{b}-\Theta_{a}+\frac{1}{2} \pi(\bmod \pi) \quad \text { in } C_{l o c}^{2}(\Omega \backslash L) \quad \text { as } \xi \rightarrow \infty, \\
P_{\xi}\left(H_{\xi} ; a, b\right)=c_{0}|L|+o(1),
\end{gathered}
$$

where $c_{0}$ is a positive constant independent of $\xi, a$ and $b,|L|$ is the length of $L$, and o(1) is a small quantity tending to zero as $\xi$ goes to infinity. Hereafter, the convergence is up to a subsequence, and we denote it as the same sequence for notation convenience.

Remark II.0. Suppose $d=1$. Then by (3.2), (3.3) and (3.10), we have

$$
u_{*}(z)=e^{\frac{1}{2} i\left(\Theta_{a}+\Theta_{b}+\frac{1}{2} \pi+h_{\xi}\right)}+o_{\xi}(1), \quad v_{*}(z)=e^{\frac{1}{2} i\left(\Theta_{a}+\Theta_{b}-\frac{1}{2} \pi+h_{\xi}\right)}+o_{\xi}(1),
$$

in $\Omega \backslash L$ as $\xi \rightarrow \infty$, where $h_{\xi}$ is a harmonic function with the admissible boundary condition. Hence $u_{*}$ and $v_{*}$ have fractional degree $1 / 2$ near $a$ and $b$, respectively. Therefore as $\epsilon \downarrow 0, \xi \uparrow \infty$, the energy minimizer may have vortices with a fractionally winding number $1 / 2$ near $a$ and $b$.

The Euler-Lagrange equation of $P_{\xi}(\cdot ; a, b)$ is

$$
\frac{1}{\sqrt{\xi}} \Delta H+\sqrt{\xi} \sin \left[2\left(\Theta_{a}-\Theta_{b}+H\right)\right]=0 \quad \text { in } \Omega .
$$

It is remarkable that $\Theta_{a}-\Theta_{b}$ is multi-valued on $L$ which is the line segment joining $a$ and $b$. As $(x, y)$ goes to $L, \Theta_{a}(x, y)-\Theta_{b}(x, y)$ may tend to $\pm \pi$, and have a $2 \pi$ gap on $L$. Since $H$ the solution of (3.12) is a single valued function, the gap of $\Theta_{a}-\Theta_{b}$ on $L$ makes $H$ have a interface around $L$. Such an interface is called a domain wall in physical literature(cf. [20]). The domain wall joins vortex centers $a$ and $b$, and the associated energy may depend on the distance of $a$ and $b$.

Proof of Theorem II: Without loss of generality, we may assume $a=(\alpha, 0), b=$ $(\beta, 0) \in \Omega$, where $\alpha$ and $\beta$ are constants such that $\alpha<\beta$. Besides, we only consider the Dirichlet boundary condition $H=\Theta_{b}-\Theta_{a}+\frac{1}{2} \pi$, on the boundary $\partial \Omega$. For the Neumann boundary condition $\partial_{\nu} H=\partial_{\nu}\left(\Theta_{b}-\Theta_{a}\right)$, one may use the similar argument to prove the theorem. To estimate the upper bound of the energy $P_{\xi}\left(H_{\xi}\right)$, we define a comparison function as follows:

$$
U_{\xi}(x, y)= \begin{cases}h_{\xi}(y) & \text { for }(x, y) \in I_{\rho, \delta} \backslash\left(B_{\rho}(a) \cup B_{\rho}(b)\right), \\ l_{a, b}(x, y) & \text { for }(x, y) \in J_{\rho, \delta} \backslash\left(B_{\rho}(a) \cup B_{\rho}(b)\right), \\ \Theta_{b}-\Theta_{a}+\frac{1}{2} \pi & \text { for }(x, y) \in \Omega \backslash\left(I_{\rho, \delta} \cup J_{\rho, \delta} \cup B_{\rho}(a) \cup B_{\rho}(b)\right), \\ h_{a, b}(x, y) & \text { for } \quad(x, y) \in B_{\rho}(a) \cup B_{\rho}(b),\end{cases}
$$


where

$$
\begin{aligned}
& I_{\rho, \delta}=\left\{(x, y): \alpha+\frac{1}{2} \rho \leq x \leq \beta-\frac{1}{2} \rho,|y| \leq \delta_{1}\right\} \\
& J_{\rho, \delta}=\left\{(x, y): \alpha+\frac{1}{2} \rho \leq x \leq \beta-\frac{1}{2} \rho, \delta_{1} \leq|y| \leq \delta_{2}\right\}
\end{aligned}
$$

$\rho=\xi^{-1 / 3}, \delta_{1}=\delta_{1}(x)=O\left(\frac{1}{6 \sqrt{2}} \frac{\log \xi}{\sqrt{\xi}}-\frac{1}{\sqrt{2}} \frac{\log \log \xi}{\sqrt{\xi}}\right)$ is determined later, and $\delta_{2}=$ $(\log \xi) / \sqrt{\xi}$. In $I_{\rho, \delta}, h_{\xi}$ is defined by $h_{\xi}(y)=h_{0}(\sqrt{\xi} y)$, where $h_{0}=h_{0}(y)$ is determined by

$$
\inf \left\{\int_{-\infty}^{\infty} \frac{1}{2}\left|h^{\prime}\right|^{2}+\cos ^{2}(h) d y: h \in H_{l o c}^{1}(\mathbf{R}), h(-\infty)=-\frac{1}{2} \pi, h(\infty)=3 \pi / 2\right\},
$$

and $h_{0}$ is the unique solution of the ordinary differential equation given by

$$
\left\{\begin{array}{l}
h^{\prime \prime}(y)=-\sin [2 h(y)], \text { for } y \in \mathbf{R}, \\
h(-\infty)=-\frac{1}{2} \pi, h(\infty)=3 \pi / 2
\end{array}\right.
$$

From the standard theorem of ordinary differential equations,

$$
\begin{aligned}
& h_{0}(y)=-\frac{1}{2} \pi+O\left(e^{-\kappa|y|}\right) \quad \text { as } y \rightarrow-\infty, \\
& h_{0}(y)=\frac{3}{2} \pi+O\left(e^{-\kappa|y|}\right) \quad \text { as } y \rightarrow \infty,
\end{aligned}
$$

where $\kappa$ is a positive constant. Moreover,

$$
\int_{-\infty}^{\infty} \frac{1}{2}\left|h_{0}^{\prime}\right|^{2}+\cos ^{2}\left(h_{0}\right) d y<\infty
$$

Since

$$
\Theta_{b}-\Theta_{a}= \begin{cases}\pi+O\left(\log \xi / \xi^{1 / 6}\right) & \text { for } \alpha+\frac{1}{2} \rho \leq x \leq \beta-\frac{1}{2} \rho, y=\delta_{2}, \\ -\pi+O\left(\log \xi / \xi^{1 / 6}\right) & \text { for } \alpha+\frac{1}{2} \rho \leq x \leq \beta-\frac{1}{2} \rho, y=-\delta_{2}\end{cases}
$$

then by (3.15) and (3.16), there exists $\delta_{1}=\delta_{1}(x)=O\left(\frac{1}{6 \kappa} \frac{\log \xi}{\sqrt{\xi}}-\frac{1}{\kappa} \frac{\log \log \xi}{\sqrt{\xi}}\right)$ such that

$$
h_{\xi}\left( \pm \delta_{1}(x)\right)=\frac{1}{2} \pi+\left(\Theta_{b}-\Theta_{a}\right)\left(x, \pm \delta_{2}\right) \quad \text { for } \alpha+\frac{1}{2} \rho \leq x \leq \beta-\frac{1}{2} \rho .
$$

It is easy to check that $\sqrt{\xi} \delta_{1}(x) \rightarrow \infty$ as $\xi \rightarrow \infty$, for $\alpha+\frac{1}{2} \rho \leq x \leq \beta-\frac{1}{2} \rho$. In $J_{\rho, \delta}$, we define $l_{a, b}$ by

$$
l_{a, b}(x, y)= \begin{cases}\frac{1}{2} \pi+\left(\Theta_{b}-\Theta_{a}\right)\left(x, \delta_{2}\right) & \text { for }(x, y) \in J_{\rho, \delta}, \delta_{1} \leq y \leq \delta_{2} \\ \frac{1}{2} \pi+\left(\Theta_{b}-\Theta_{a}\right)\left(x,-\delta_{2}\right) & \text { for }(x, y) \in J_{\rho, \delta},-\delta_{2} \leq y \leq-\delta_{1}\end{cases}
$$

Then

$$
\int_{J_{\rho, \delta}} \frac{1}{4 \sqrt{\xi}}\left|\nabla l_{a, b}\right|^{2}+\frac{1}{2} \sqrt{\xi} \cos ^{2}\left(\Theta_{a}-\Theta_{b}+l_{a, b}\right)=o_{\xi}(1) .
$$

In $B_{\rho}(a) \cup B_{\rho}(b)$, we let $h_{a, b}$ be the harmonic function with admissible boundary conditions that

$$
h_{a, b}= \begin{cases}h_{\xi} & \text { on } \partial\left(B_{\rho}(a) \cup B_{\rho}(b)\right) \cap I_{\rho, \delta} \\ l_{a, b} & \text { on } \partial\left(B_{\rho}(a) \cup B_{\rho}(b)\right) \cap J_{\rho, \delta} \\ \Theta_{b}-\Theta_{a}+\frac{1}{2} \pi & \text { on } \partial\left(B_{\rho}(a) \cup B_{\rho}(b)\right) \backslash\left(I_{\rho, \delta} \cup J_{\rho, \delta}\right) .\end{cases}
$$


Then

$$
\int_{B_{\rho}(a) \cup B_{\rho}(b)} \frac{1}{4 \sqrt{\xi}}\left|\nabla h_{a, b}\right|^{2}+\frac{1}{2} \sqrt{\xi} \cos ^{2}\left(\Theta_{a}-\Theta_{b}+h_{a, b}\right)=o_{\xi}(1) .
$$

By (3.18), (3.19) and the definition of $h_{a, b}$, we have $U_{\xi} \in H^{1}(\Omega ; \mathbb{R})$. Moreover, by (3.17), (3.20) and (3.21), we have

$$
P_{\xi}\left(U_{\xi} ; a, b\right)=c_{0}(\beta-\alpha)+o_{\xi}(1)
$$

where $c_{0}$ is a positive constant from (3.17). Thus

$$
P_{\xi}\left(H_{\xi} ; a, b\right) \leq P_{\xi}\left(U_{\xi} ; a, b\right)=c_{0}(\beta-\alpha)+o_{\xi}(1)
$$

By (3.22), we have

$$
H_{\xi}+\Theta_{a}-\Theta_{b} \rightarrow \frac{1}{2} \pi \quad \text { almost everywhere as } \xi \rightarrow \infty
$$

Such a convergence may be up to a subsequence. For notation convenience, we may use the same sequence to denote it in the rest of this paper. Please note that $\Theta_{a}-\Theta_{b}$ is well-defined only in $\Omega \backslash L$, where $L=\{(x, 0): \alpha \leq x \leq \beta\}$ is the line segment joining $a$ and $b$. We may define $\tilde{H}_{\xi}=H_{\xi}+\Theta_{a}-\Theta_{b}$ in $\Omega \backslash L$. Then $\tilde{H}_{\xi}$ satisfies

$$
\frac{1}{\sqrt{\xi}} \Delta \tilde{H}_{\xi}+\sqrt{\xi} \sin \left(2 \tilde{H}_{\xi}\right)=0 \quad \text { in } \Omega \backslash L .
$$

The equation (3.24) is a standard singular perturbation problem(cf. [1], [15], [21], etc). Hence by (3.23) and (3.24), we have

$$
H_{\xi}+\Theta_{a}-\Theta_{b} \rightarrow \frac{1}{2} \pi \quad \text { exponentially in } \Omega \backslash L \text { as } \xi \rightarrow \infty,
$$

and (3.10) holds. Let $\hat{H}(x, \hat{y})=H_{\xi}(x, \hat{y} / \sqrt{\xi})$. Then by $(3.25)$, we have

$$
\begin{aligned}
P_{\xi}\left(H_{\xi} ; a, b\right) & \geq \int_{\alpha+\sigma}^{\beta-\sigma} \int_{-\xi^{-1 / 3}}^{\xi^{-1 / 3}} \frac{1}{4 \sqrt{\xi}}\left|\nabla H_{\xi}\right|^{2}+\frac{1}{2} \sqrt{\xi} \cos ^{2}\left(\Theta_{a}-\Theta_{b}+H_{\xi}\right) d y d x \\
& \geq \int_{\alpha+\sigma}^{\beta-\sigma} \int_{-\xi^{1 / 6}}^{\xi^{1 / 6}} \frac{1}{4}\left|\partial_{\hat{y}} \hat{H}\right|^{2}+\frac{1}{2} \cos ^{2}\left(\Theta_{a}-\Theta_{b}+\hat{H}\right) d \hat{y} d x \\
& =\int_{\alpha+\sigma}^{\beta-\sigma} \int_{-\xi^{1 / 6}}^{\xi^{1 / 6}} \frac{1}{4}\left|\partial_{\hat{y}} \hat{H}\right|^{2}+\frac{1}{2} \cos ^{2}(\hat{H}) d \hat{y} d x+o(1) \\
& \geq c_{0}[(\beta-\alpha)-2 \sigma]+o_{\xi}(1),
\end{aligned}
$$

i.e.

$$
P_{\xi}\left(H_{\xi} ; a, b\right) \geq c_{0}[(\beta-\alpha)-2 \sigma]+o_{\xi}(1),
$$

for $\sigma>0$, where $c_{0}$ is a positive constant from (3.17). Here we have used the fact that $\Theta_{a}-\Theta_{b}= \pm \pi+O\left(\xi^{-1 / 3}\right)$ for $\alpha+\sigma \leq x \leq \beta-\sigma,|y|=\xi^{-1 / 3}$. Consequently, we have the lower bound estimate

$$
P_{\xi}\left(H_{\xi} ; a, b\right) \geq c_{0}(\beta-\alpha)+o_{\xi}(1)
$$


by letting $\sigma$ go to zero. Therefore we may complete the proof of Theorem II.

Remark II.1. We may generalize Theorem II to the case that

$$
a=\left(a_{1}, \cdots, a_{d}\right), \quad b=\left(b_{1}, \cdots, b_{d}\right) \in \Omega^{d},
$$

and $a_{j}$ 's and $b_{k}$ 's are distinct $2 d$ points. As for Theorem II, there are $d$ line segments $L_{j}=\left[a_{k_{j}}, b_{k_{j}}\right], j=1, \cdots, d$ joining these $2 d$ points, where $\left[a_{k_{j}}, b_{k_{j}}\right]$ denotes the line segment joining $a_{k_{j}}$ and $b_{k_{j}}$. Due to the energy minimization, the line segments $L_{j}$ 's must have the smallest length of all $d$ line segments joining $a_{j}$ 's and $b_{k}$ 's in $d$ pairs.

3.1. Remarks on energy minimization for $1<<\xi<<\log \frac{1}{\epsilon}$.

In this section, we state three cases on the energy minimization problem of the energy functional (1.4) for $1<<\xi<<\log \frac{1}{\epsilon}$. Two cases of them can be analyzed by Theorem I and II. However, the rest of the cases are open and would be very difficult. Now we state these cases as follows:

Good case. No domain walls:

Assume the boundary conditions $\left.u^{1}\right|_{\partial \Omega}=g_{1},\left.u^{2}\right|_{\partial \Omega}=g_{2}$ satisfying $g_{1} \cdot g_{2}=0$ i.e.,

$$
g_{1}=e^{ \pm i \frac{\pi}{2}} g_{2}
$$

In this case one can show: If $\left(u_{\epsilon, \xi}^{1}, u_{\epsilon, \xi}^{2}\right)$ are minimizers of (1.4) such that $u_{\epsilon, \xi}^{1}=$ $g_{1}, u_{\epsilon, \xi}^{2}=g_{2}$ on $\partial \Omega$. Then

$$
\begin{aligned}
E_{\epsilon, \xi}\left(u_{\epsilon, \xi}^{1}, u_{\epsilon, \xi}^{2}\right) & =\left(2 \pi \log \frac{1}{\epsilon}\right) \cdot d+O(1) \\
& =2 \pi d \log \frac{1}{\epsilon}+W\left(a, g^{1}, \Omega\right)+o_{\epsilon, \xi}(1)
\end{aligned}
$$

Here $d=\left|\operatorname{deg} g_{1}\right|$, and the renormalized energy $W$ reaches minimun at $a$.

Indeed,one may simply choose $d$ points $b^{1}, \ldots, b^{d} \in \Omega$, and one constructs maps $V_{\epsilon, \xi}^{1}, V_{\epsilon, \xi}^{2}$ with vortices at $b^{1}, \ldots, b^{d}$ such that $V_{\epsilon, \xi}^{j}$ 's have no domain walls, and

$$
E_{\epsilon, \xi}\left(V_{\epsilon, \xi}^{1}, V_{\epsilon, \xi}^{2}\right)=2 \pi d \log \frac{1}{\epsilon}+O_{\epsilon, \xi}(1)
$$

Here one notices that $H \equiv \frac{\pi}{2}$ in $\Omega$ and $h$ is harmonic. From (3.27), one has

$$
E_{\epsilon, \xi}\left(u_{\epsilon, \xi}^{1}, u_{\epsilon, \xi}^{2}\right) \leq 2 \pi d \log \frac{1}{\epsilon}+O(1) .
$$

By Theorem I and II, we may conclude that vortices of $u_{\epsilon, \xi}^{1}$ and $u_{\epsilon, \xi}^{2}$ have to be at essentially the same locations. Since if vortex locations for two components are different by a fixed amount, then $H \equiv \pm \frac{\pi}{2}$ in $\Omega$ a.e. would imply $H$ has to have jumps across some hypersurfaces i.e. $H \not \equiv \frac{\pi}{2}$, the region $H=\frac{\pi}{2}$ and $H=-\frac{\pi}{2}$ both are nontrivial. Then it is not hard to see $E_{\epsilon, \xi}\left(u_{\epsilon, \xi}^{1}, u_{\epsilon, \xi}^{2}\right) \geq 2 \pi \log \frac{1}{\epsilon}+O(\sqrt{\xi})$. This would contradict the energy upper bound if $\xi$ is sufficiently large.

Bad case 1. Domain wall is simply a line connecting end points of $\Gamma_{1}$ :

Here we assume $\Gamma_{1} \subseteq \partial \Omega$ a smooth connected curve such that the line segment joining endpoints of $\Gamma_{1}$ is contained in $\Omega$. Now we assume the boundary conditions satisfying

$$
\begin{aligned}
& g_{1}=e^{i \frac{\pi}{2}} g_{2} \text { on } \Gamma_{1} \\
& g_{1}=e^{-i \frac{\pi}{2}} g_{2} \text { on } \quad \partial \Omega \backslash \Gamma_{1} .
\end{aligned}
$$


In this case there has to be a domain wall joining endpoints of $\Gamma_{1}$, that is, subregions of $H$ to be $\frac{\pi}{2}$ and $-\frac{\pi}{2}$ are both nontrivial. But since the degrees of $g_{1}$ and $g_{2}$ are the same, one may smooth $g_{1}$ to avoid jump near endpoints of $\Gamma_{1}$. Thus one can construct a comparison $\left(V^{1}, V^{2}\right)$ s.t.

$$
\begin{aligned}
E_{\epsilon, \xi}\left(V^{1}, V^{2}\right) & =2 \pi d \log \frac{1}{\epsilon}+c_{0} l_{1} \sqrt{\xi}+O(1) \\
& =2 \pi d \log \frac{1}{\epsilon}(1+o(1))
\end{aligned}
$$

Here we have used the assumption $1<<\xi<<\log \frac{1}{\epsilon}$, and $l_{1}=$ length of the segment connecting endpoints of $\Gamma_{1}$. On the other hand, by the first two integrals of the energy functional (1.4), it is easy to see

$$
E_{\epsilon, \xi}\left(u_{\epsilon, \xi}^{1}, u_{\epsilon, \xi}^{2}\right) \geq 2 \pi d \log \frac{1}{\epsilon}+O(1) .
$$

Thus the minimizing map has exactly $d$ vortices for each component. Suppose when $\sqrt{\xi} \rightarrow+\infty,(\epsilon \rightarrow 0)$ these vortices do not come together. Then as for the Good case described above, we may get contradiction by energy comparisons as there would be some extra domain walls. Furthermore, by Theorem II and energy asymptotic expansion, we have

$$
E_{\epsilon, \xi}\left(u_{\epsilon, \xi}^{1}, u_{\epsilon, \xi}^{2}\right)=2 \pi d \log \frac{1}{\epsilon}+c_{0} l_{1} \sqrt{\xi}+O(1) .
$$

Bad case 2. Assume $\operatorname{deg} g_{1}=\operatorname{deg} g_{2}$, and both $g^{\prime} s$ are smooth but $g_{1} \cdot g_{2} \neq 0$ on $\partial \Omega$ :

Since the minimum energy upper bound $\leq 2 \pi d \log \frac{1}{\epsilon}(1+\delta), \forall \delta>0$ it is obvious that $\log \frac{1}{\epsilon}>>\xi$. This already implies that both $u_{\epsilon, \xi}^{1}$ and $u_{\epsilon, \xi}^{2}$ have exactly $d$ "essential zeros" or "vortices". However, the minimum energy for the phase functions could be very high and there could be another type(not due to topological reasons) of boundary layer to form since $H$ takes limit values $\frac{\pi}{2}$ or $-\frac{\pi}{2}$. We also note that from [2], it was found that there exists a mapping between a two-flavor Ginzburg-Landau functional and a version of a $O(3)$-symmetric Faddeev model. This implies in particular that such a system possesses a hidden $O(3)$ symmetry and allows for the formation of stable knotted solitons for the phase functions. The knotted solitons are much more complex and structurally complicated topological defects than Abrikosov vortices, and thus its realization in superconductors should open an exceptionally wide range of possibilities of studies of various phenomena associated with them.

\section{Dynamics of Domain Walls}

Now we study the dynamics of vortices connected by domain walls governed by a system of coupled Ginzburg-Landau equations given by

$$
\begin{array}{ll}
\frac{1}{\lambda_{\epsilon, \xi}} u_{t}=\Delta u+\frac{1}{\epsilon^{2}}\left(1-|u|^{2}\right) u-\xi(u \cdot v) v & \text { in } \Omega \times \mathbf{R}_{+}, \\
\frac{1}{\lambda_{\epsilon, \xi}} v_{t}=\Delta v+\frac{1}{\epsilon^{2}}\left(1-|v|^{2}\right) v-\xi(u \cdot v) u & \text { in } \Omega \times \mathbb{R}_{+},
\end{array}
$$

with the Neumann boundary conditions and initial conditions as follows:

$$
\begin{gathered}
\partial_{\nu} u(x, t)=\partial_{\nu} v(x, t)=0, \text { for } x \in \partial \Omega, t \geq 0, \\
u(x, 0)=U_{\epsilon, \xi}^{0}(x), \text { for } x \in \Omega \\
v(x, 0)=V_{\epsilon, \xi}^{0}(x), \text { for } x \in \Omega
\end{gathered}
$$


The solution $(u, v)$ of (4.1)-(4.5) may depend on $\epsilon$ and $\xi$. For simplicity, we only use $(u, v)$ to denote it. Here $\Omega$ is a two-dimensional, smooth, bounded domain, $\epsilon, \xi$ and $\lambda_{\epsilon, \xi}$ are positive parameters, and $(u, v): \Omega \times \mathbf{R}_{+} \rightarrow \mathbb{C}^{2}$ is smooth.

For the initial data $U_{\epsilon, \xi}^{0}(x)$ and $V_{\epsilon, \xi}^{0}(x)$, we make the following assumptions:

$\left(A_{1}\right)$

$$
\mid \begin{aligned}
& U_{\epsilon, \xi}^{0}(x)|, \quad| \begin{array}{l}
V_{\epsilon, \xi}^{0}(x) \\
\nabla U_{\epsilon, \xi}^{0}(x)
\end{array}|, \quad| \nabla V_{\epsilon, \xi}^{0}(x) \mid \leq \frac{K}{\epsilon} \sqrt{\xi}, \quad \text { for } x \in \Omega,
\end{aligned}
$$

where $K$ is a positive constant independent of $\epsilon$ and $\xi$,

$\left(A_{2}\right) \quad$ For all $\xi>0$, as $\epsilon \rightarrow 0+$,

$$
\begin{gathered}
U_{\epsilon, \xi}^{0}(x) \rightarrow \frac{x-a}{|x-a|} e^{i \phi_{\xi}^{0}(x)} \quad \text { weakly in } H_{l o c}^{1}(\bar{\Omega} \backslash\{a\}), \\
V_{\epsilon, \xi}^{0}(x) \rightarrow \frac{x-b}{|x-b|} e^{i \Phi_{\xi}^{0}(x)} \quad \text { weakly in } H_{l o c}^{1}(\bar{\Omega} \backslash\{b\}),
\end{gathered}
$$

where $a$ and $b$ are two distinct points in $\Omega$, and $\phi_{\xi}^{0}$ and $\Phi_{\xi}^{0}$ are of $H^{1}(\Omega ; \mathbf{R})$,

$\left(A_{3}\right)$

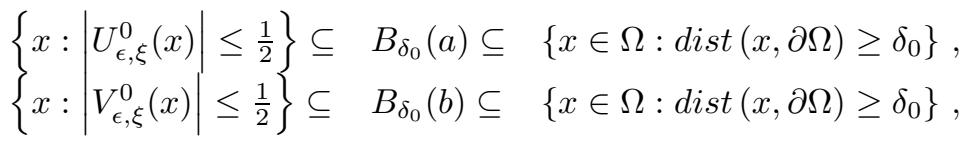

where $\delta_{0}$ is a positive constant depending only on points $a, b$ and the domain $\Omega$ such that $B_{\delta_{0}}(a)$ and $B_{\delta_{0}}(b)$ are two disjoint balls in $\Omega$,

$\left(A_{4}\right) \quad$ For $\xi>0$,

$$
E_{\epsilon, \xi}\left(U_{\epsilon, \xi}^{0}, V_{\epsilon, \xi}^{0}\right) \leq 2 \pi \log \frac{1}{\epsilon}+C_{\xi}, \quad \text { as } \epsilon \rightarrow 0+,
$$

where $C_{\xi}$ is a positive constant depending on $\xi$.

$\left(A_{5}\right) \quad$ As $\xi \rightarrow+\infty, \phi_{\xi}^{0}-\Phi_{\xi}^{0}$ forms a domain wall $L^{0}$ which is a line segment joining points $a$ and $b$, and satisfies

$$
P_{\xi}\left(\phi_{\xi}^{0}-\Phi_{\xi}^{0} ; a, b\right)=c_{0}\left|L^{0}\right|+o_{\xi}(1) \quad \text { as } \xi \rightarrow+\infty,
$$

where $P_{\xi}$ is defined in (3.7), $\left|L^{0}\right|$ is the length of $L^{0}$, and $c_{0}$ (defined in (3.11)) is a positive constant independent of $\xi, a$ and $b$,

$\left(A_{6}\right)$

$$
\lim _{\epsilon \rightarrow 0+}\left[E_{\epsilon, \xi}\left(U_{\epsilon, \xi}^{0}, V_{\epsilon, \xi}^{0}\right)-2 \pi \log \frac{1}{\epsilon}\right]=\sqrt{\xi}\left(c_{0}\left|L^{0}\right|+o_{\xi}(1)\right),
$$

as $\xi \rightarrow+\infty$, where $E_{\epsilon, \xi}$ is defined in (3.1), and $|\Omega|$ is the volume of $\Omega$, and $o_{\xi}(1)$ is a small quantity which tends to zero as $\xi \rightarrow+\infty$. 
The system of equations (4.1) and (4.2) may arise in the gradient flow of the energy functional $E_{\epsilon, \xi}(u, v)$ given by

$$
\frac{1}{\lambda_{\epsilon, \xi}}(u, v)_{t}=-\frac{\delta E_{\epsilon, \xi}(u, v)}{\delta(u, v)}
$$

where $E_{\epsilon, \xi}(u, v)$ is defined in (3.1), and $\lambda_{\epsilon, \xi}$ is a positive constant for time scale depending only on $\epsilon$ and $\xi$.

As $\xi=0$, the problem (4.1)-(4.5) becomes a standard Ginzburg-Landau equation, and the vortex dynamics is well-known (cf. [11] and [12]). Basically, vortices are static when the time scale is of $O(1)$, and vortices start to move and the motion is governed by a system of ordinary differential equations when the time scale is of $O(-\log \epsilon)$. In this section, we assume $-\log \epsilon \gg \xi \gg 1$ and find out the different dynamic law of vortices due to the effect of domain walls. When the time scale is of order $O(1)$, vortices are still static. In the time scale of order $O(\sqrt{\xi})$, the curved domain walls do a motion by the mean curvature while fractional degree vortices located at the boundaries of domain walls are essentially static. Furthermore, in the time scale of order $O(-(\log \epsilon) / \sqrt{\xi})$, the domain wall has become a line segment and starts to shorten itself in constant speed, and pulls vortices at the ends of the domain wall to be a pair. Such a new vortex dynamic law is illustrated by two theorems. One is for the time scale of order $O(1)$, and the other is for the time scale of order $O(-(\log \epsilon) / \sqrt{\xi})$. For such a time scale, first we let $\epsilon$ tend to zero and fix $\xi$ as any constant, and then let $\xi$ tend to $\infty$. Now we state these two theorems as follows:

Theorem III. Let $\left(U_{\epsilon, \xi}(x, t), V_{\epsilon, \xi}(x, t)\right)$ be the solution of (4.1)-(4.5) with $\left(U_{\epsilon, \xi}^{0}(x)\right.$, $\left.V_{\epsilon, \xi}^{0}(x)\right)$ satisfying the assumptions $\left(A_{1}\right)-\left(A_{4}\right)$. Then we have the following conclusions:

$$
\begin{aligned}
& \text { If } \lambda_{\epsilon, \xi} \equiv 1 \text {, then } \\
& \qquad \begin{aligned}
U_{\epsilon, \xi}(x, t) & \rightarrow \frac{x-a}{|x-a|} e^{i \phi_{\xi}(x, t)} \quad \text { weakly in } H_{l o c}^{1}(\bar{\Omega} \backslash\{a\}), \\
V_{\epsilon, \xi}(x, t) & \rightarrow \frac{x-b}{|x-b|} e^{i \Phi_{\xi}(x, t)} \quad \text { weakly in } H_{l o c}^{1}(\bar{\Omega} \backslash\{b\}),
\end{aligned}
\end{aligned}
$$

for each $\xi, t>0$ as $\epsilon \rightarrow 0+$, where $\left(\phi_{\xi}(x, t), \Phi_{\xi}(x, t)\right)$ is the solution of the problem

$$
\left\{\begin{array}{lll}
\partial_{t} h_{\xi} & =\Delta h_{\xi} & \text { in } \Omega \times \mathbb{R}_{+}, \\
\partial_{t} H_{\xi} & =\Delta H_{\xi}+\xi \sin \left[2\left(\Theta_{a}-\Theta_{b}+H_{\xi}\right)\right] & \text { in } \Omega \times \mathbb{R}_{+}, \\
\partial_{\nu} \phi_{\xi} & =-\partial_{\nu} \Theta_{a} & \text { on } \partial \Omega, \\
\partial_{\nu} \Phi_{\xi} & =-\partial_{\nu} \Theta_{b} & \text { on } \partial \Omega, \\
\phi_{\xi}(x, 0) & =\phi^{0}(x) & \text { for } x \in \Omega, \\
\Phi_{\xi}(x, 0) & =\Phi^{0}(x) & \text { for } x \in \Omega,
\end{array}\right.
$$

where $h_{\xi}=\phi_{\xi}+\Phi_{\xi}$ and $H_{\xi}=\phi_{\xi}-\Phi_{\xi}$.

(ii) If $\lambda_{\epsilon, \xi} / \sqrt{\xi} \rightarrow+\infty$ and $\sqrt{\xi} \lambda_{\epsilon, \xi} / \log \frac{1}{\epsilon} \rightarrow 0$ as $\log (1 / \epsilon) \gg \xi \rightarrow \infty$, 
then

$$
\begin{aligned}
U_{\epsilon, \xi}(x, t) & \rightarrow \frac{x-a}{|x-a|} e^{i \phi_{\xi}(x)}, \quad \text { in } L_{l o c}^{2}\left(\bar{\Omega} \times \mathbb{R}_{+}\right), \\
V_{\epsilon, \xi}(x, t) & \rightarrow \frac{x-b}{|x-b|} e^{i \Phi_{\xi}(x)} \quad \text { in } L_{l o c}^{2}\left(\bar{\Omega} \times \mathbf{R}_{+}\right), \\
U_{\epsilon, \xi}(x, t) & \rightarrow \frac{x-a}{|x-a|} e^{i \phi_{\xi}(x)} \quad \text { weakly in } H_{l o c}^{1}(\bar{\Omega} \backslash\{a\}), \\
V_{\epsilon, \xi}(x, t) & \rightarrow \frac{x-b}{|x-b|} e^{i \Phi_{\xi}(x)} \quad \text { weakly in } H_{l o c}^{1}(\bar{\Omega} \backslash\{b\}),
\end{aligned}
$$

for $t>0$, where $\left(\phi_{\xi}(x, t), \Phi_{\xi}(x, t)\right)$ is the solution of the problem

$$
\left\{\begin{array}{ccc}
\Delta h_{\xi} & =0 & \text { in } \Omega, \\
\Delta H_{\xi} & =-\xi \sin \left[2\left(\Theta_{a}-\Theta_{b}+H_{\xi}\right)\right] & \text { in } \Omega, \\
\partial_{\nu} \phi_{\xi} & =-\partial_{\nu} \Theta_{a} & \text { on } \partial \Omega, \\
\partial_{\nu} \Phi_{\xi} & =-\partial_{\nu} \Theta_{b} & \text { on } \partial \Omega,
\end{array}\right.
$$

where $h_{\xi}=\phi_{\xi}+\Phi_{\xi}$ and $H_{\xi}=\phi_{\xi}-\Phi_{\xi}$.

Proof of Theorem III: The proof is identical to that for Theorem 3.7 in [10] and Theorem 2.1 (i) and (ii) in [12]. The only difference is the sine-Gordon type equations (4.6) and (4.7) of the phase function $H_{\xi}$. We may explain that as follows: By Proposition 1.2 of [13], as $\epsilon \rightarrow 0+$ (up to a subsequence), $U_{\epsilon, \xi}$ and $V_{\epsilon, \xi}$ have the limit functions $U_{0, \xi}$ and $V_{0, \xi}$ with the form

$$
U_{0, \xi}(x, t)=e^{i\left(\Theta_{a}+\phi_{\xi}\right)}, \quad V_{0, \xi}(x, t)=e^{i\left(\Theta_{b}+\Phi_{\xi}\right)} .
$$

Now we take the wedge product for $u$ and the equation (4.1), $v$ and the equation (4.2) and set $u=U_{\epsilon, \xi}$ and $v=V_{\epsilon, \xi}$. Hence by the same argument of pp. 335 and Theorem 3.7 in [10], we may obtain the equations (4.6) and (4.7), respectively, and complete the proof of Theorem III.

Remark III. Under the assumptions of Theorem III, suppose as $\xi \rightarrow+\infty, \phi_{\xi}^{0}-\Phi_{\xi}^{0}$ forms a curved domain wall $\gamma^{0}$ which may be a smooth curve. Then by the system (4.6) and results of [8] Section 12.2, one may show that in the time scale of order $O(\sqrt{\xi})$ with proper initial data, curved domain walls do a motion by the mean curvature while fractional degree vortices located at the boundaries of domain walls are essentially static. Here the condition $1 \ll \sqrt{\xi} \ll\left(\log \frac{1}{\epsilon}\right) / \sqrt{\xi}$ is crucial to show boundaries of domain walls are essentially static.

Now we consider the time scale is of order $O\left(\left(\log \frac{1}{\epsilon}\right) / \sqrt{\xi}\right)$ and see the effect of $\xi$ on the dynamics of vortices. For this purpose we first define the renormalized energy for the case of the Neumann boundary condition. Let $a$ and $b$ be two distinct points in $\Omega$, and let

$$
\begin{aligned}
& U_{a}(x)=\frac{x-a}{|x-a|} e^{i h_{a}(x)}, \quad \text { for } x \in \Omega, \\
& V_{b}(x)=\frac{x-b}{|x-b|} e^{i h_{b}(x)}, \quad \text { for } x \in \Omega .
\end{aligned}
$$

Here $h_{a}(x)$ and $h_{b}(x)$ are functions defined later on the whole $\Omega$ with

$$
\partial_{\nu} h_{a}=-\partial_{\nu} \Theta_{a}, \quad \text { and } \partial_{\nu} h_{b}=-\partial_{\nu} \Theta_{b}
$$


on the boundary $\partial \Omega$. We define the renormalized energy by

$$
W(a, b ; \xi)=\lim _{\rho \downarrow 0}\left[\int_{\Omega \backslash\left(B_{\rho}(a) \cup B_{\rho}(b)\right)} \begin{array}{l}
\frac{1}{2}\left|\nabla U_{a}\right|^{2}+\frac{1}{2}\left|\nabla V_{b}\right|^{2} \\
+\frac{1}{2} \xi\left(U_{a} \cdot V_{b}\right)^{2} d x d y-2 \pi \log \frac{1}{\rho}
\end{array}\right] .
$$

It is easy to check that (4.10) is well-defined and equal to

$$
\begin{aligned}
& \lim _{\rho \downarrow 0}\left[\int_{\Omega \backslash B_{\rho}(a)} \frac{1}{2}\left|\nabla \Theta_{a}\right|^{2} d x-\pi \log \frac{1}{\rho}\right] \\
+ & \lim _{\rho \downarrow 0}\left[\int_{\Omega \backslash B_{\rho}(b)} \frac{1}{2}\left|\nabla \Theta_{b}\right|^{2} d x-\pi \log \frac{1}{\rho}\right] \\
+ & \int_{\Omega} \frac{1}{2}\left(\left|\nabla h_{a}\right|^{2}+\left|\nabla h_{b}\right|^{2}\right)+\frac{1}{2} \xi \cos ^{2}\left(\Theta_{a}-\Theta_{b}+h_{a}-h_{b}\right) .
\end{aligned}
$$

The third integral can be written as

$$
\int_{\Omega} \frac{1}{4}\left|\nabla h_{a, b}\right|^{2}+\int_{\Omega} \frac{1}{4}\left|\nabla H_{a, b}\right|^{2}+\frac{1}{2} \xi \cos ^{2}\left(\Theta_{a}-\Theta_{b}+H_{a, b}\right)
$$

where $H_{a, b}(x)=h_{a}(x)-h_{b}(x)$ and $h_{a, b}=h_{a}(x)+h_{b}(x)$. To minimize such an integral, we set $h_{a, b}$ as a harmonic function, and $H_{a, b}$ is the energy minimizer of the energy functional

$$
\int_{\Omega} \frac{1}{4}|\nabla H|^{2}+\frac{1}{2} \xi \cos ^{2}\left(\Theta_{a}-\Theta_{b}+H\right)
$$

with

$$
\partial_{\nu} h_{a, b}=-\partial_{\nu}\left(\Theta_{a}+\Theta_{b}\right), \quad \partial_{\nu} H_{a, b}=-\partial_{\nu}\left(\Theta_{a}-\Theta_{b}\right)
$$

on the boundary $\partial \Omega$. Note that

$$
\begin{aligned}
& \int_{\partial \Omega} \partial_{\nu} h_{a, b}=-\int_{\partial \Omega} \partial_{\nu}\left(\Theta_{a}+\Theta_{b}\right)=\int_{\partial B_{r}(a)} \partial_{\nu}\left(\Theta_{a}+\Theta_{b}\right)=0 \\
& \int_{\partial \Omega} \partial_{\nu} H_{a, b}=-\int_{\partial \Omega} \partial_{\nu}\left(\Theta_{a}-\Theta_{b}\right)=\int_{\partial B_{r}(b)} \partial_{\nu}\left(\Theta_{a}-\Theta_{b}\right)=0
\end{aligned}
$$

(for all sufficiently small $r>0)$ and hence such $h_{a, b}(x)$ (up to constants) and $H_{a, b}(x)(\bmod$ $\pi)$ exist. As $\xi \rightarrow \infty$, by Theorem II, the domain wall $L$ comes out, and the second integral of (4.11) becomes

$$
\begin{aligned}
& \int_{\Omega} \frac{1}{4}\left|\nabla H_{a, b}\right|^{2}+\frac{1}{2} \xi \cos ^{2}\left(\Theta_{a}-\Theta_{b}+H_{a, b}\right) d x \\
& =\sqrt{\xi}\left[c_{0}|L|+o_{\xi}(1)\right],
\end{aligned}
$$

where $c_{0}$ is the positive constant defined in Theorem II, $L$ is the line segment joining $a$ and $b,|L|$ is the length of $L$, and $o_{\xi}(1)$ is a small quantity tending to zero as $\xi$ goes to infinity. Hence the renormalized energy satisfies

$$
W(a, b ; \xi)=\sqrt{\xi}\left[c_{0}|L|+o_{\xi}(1)\right] \quad \text { as } \xi \rightarrow+\infty .
$$

It is well-known that the renormalized energy is crucial to the dynamics of vortices. Hence the dynamics of the domain wall may dominate the dynamics of vortices. This may result in new dynamics of vortices. 
The main result of this section is

Theorem IV. Under the same assumptions of Theorem III and the assumptions $\left(A_{5}\right)$ and $\left(A_{6}\right)$, if $\lambda_{\epsilon, \xi}=\left(\log \frac{1}{\epsilon}\right) / \sqrt{\xi}$, then

$$
\begin{aligned}
& U_{\epsilon, \xi}(x, t) \rightarrow \frac{x-a_{\xi}(t)}{\left|x-a_{\xi}(t)\right|} e^{i \phi_{\xi}(x)} \quad \text { weakly in } H_{l o c}^{1}\left(\bar{\Omega} \backslash\left\{a_{\xi}(t)\right\}\right), \\
& V_{\epsilon, \xi}(x, t) \rightarrow \frac{x-b_{\xi}(t)}{\left|x-b_{\xi}(t)\right|} e^{i \Phi_{\xi}(x)} \quad \text { weakly in } H_{l o c}^{1}\left(\bar{\Omega} \backslash\left\{b_{\xi}(t)\right\}\right),
\end{aligned}
$$

for each $\xi>0$ and $0 \leq t \leq T$ as $\epsilon \rightarrow 0+$, where $a_{\xi}(t)$ and $b_{\xi}(t)$ are two distinct points in $\Omega,\left(\phi_{\xi}(x, t), \Phi_{\xi}(x, t)\right)$ is the solution of the problem

$$
\left\{\begin{array}{ccc}
\Delta h_{\xi} & =0 & \text { in } \Omega, \\
\Delta H_{\xi}=-\xi \sin \left[2\left(\Theta_{a_{\xi}(t)}-\Theta_{b_{\xi}(t)}+H_{\xi}\right)\right] & \text { in } \Omega, \\
\partial_{\nu} \phi_{\xi} & =-\partial_{\nu} \Theta_{a_{\xi}(t)} & \text { on } \partial \Omega, \\
\partial_{\nu} \Phi_{\xi} & =-\partial_{\nu} \Theta_{b_{\xi}(t)} & \text { on } \partial \Omega,
\end{array}\right.
$$

where $h_{\xi}=\phi_{\xi}+\Phi_{\xi}$ and $H_{\xi}=\phi_{\xi}-\Phi_{\xi} \cdot A s \xi \rightarrow+\infty$ (up to a subsequence), $a_{\xi}(t)$ and $b_{\xi}(t)$ converge uniformly to $a(t)$ and $b(t)$ on a finite time interval $[0, T]$, respectively. Moreover, $a(t)$ and $b(t)$ satisfy

$$
\frac{d}{d t} a(t)=c_{1} \overrightarrow{a b}, \quad \frac{d}{d t} b(t)=-c_{1} \overrightarrow{a b}
$$

where $c_{1}$ is a positive constant depending only on the constant $c_{0}$ defined in (3.17), and $\overrightarrow{a b}$ is the unit vector from the point a to the point $b$.

Remark IV.1. The motion equation (4.16) shows that the line segment $L$ with finite length $|a-b|$ starts to shorten itself in a constant speed. Since the equation (4.16) holds when vortices $a$ and $b$ are separate, then the length of the finite interval $T$ must satisfy $T<\frac{1}{2}|a-b| / c_{1}$.

Proof of Theorem IV: As $\xi$ is positive and finite, we may follow the same idea of the proof of Theorem 2.1 (iii) in [12], and we may obtain (4.13)-(4.15). Now we may sketch the proof as follows:

Step 1. We define

$$
\lambda_{\epsilon, \xi} \mu_{\epsilon, \xi}(t)=\left\{\begin{array}{l}
\frac{1}{2}\left(\left|\nabla U_{\epsilon, \xi}\right|^{2}+\left|\nabla V_{\epsilon, \xi}\right|^{2}\right) \\
+\frac{1}{4 \epsilon^{2}}\left[\left(1-\left|U_{\epsilon, \xi}\right|^{2}\right)^{2}+\left(1-\left|V_{\epsilon, \xi}\right|^{2}\right)^{2}\right] \\
+\frac{1}{2} \xi\left(U_{\epsilon, \xi} \cdot V_{\epsilon, \xi}\right)^{2}
\end{array}\right\} d x d y
$$

for $t \in\left[0, T_{\epsilon, \xi}\right]$, where $T_{\epsilon, \xi}$ is defined as in the proof of Theorem 2.1 (iii) in [12]. Then $\mu_{\epsilon, \xi}(t)$ is a family, $0<\epsilon<1$, of bounded Radon measure for $t \in\left[0, T_{\epsilon, \xi}\right]$ and $\xi>0$. 
Moreover, by (4.1) and (4.2), we may calculate

$$
\begin{aligned}
\frac{d}{d t} & \int_{\Omega} \phi^{2}(x) \mu_{\epsilon, \xi}(t) d x \\
= & -\lambda_{\epsilon, \xi}^{-2} \int_{\Omega} \phi^{2}\left(\left|\frac{\partial U_{\epsilon, \xi}}{\partial t}\right|^{2}+\left|\frac{\partial V_{\epsilon, \xi}}{\partial t}\right|^{2}\right) d x \\
& -2 \lambda_{\epsilon, \xi}^{-1} \int_{\Omega} \phi \nabla \phi \cdot\left(\nabla U_{\epsilon, \xi} \frac{\partial U_{\epsilon, \xi}}{\partial t}+V_{\epsilon, \xi} \frac{\partial V_{\epsilon, \xi}}{\partial t}\right) d x \\
\leq & \int_{\Omega} \phi^{2} \mu_{\epsilon, \xi}(t) d x+\lambda_{\epsilon, \xi}^{-1} C(\phi) \int_{\Omega}\left|\frac{\partial U_{\epsilon, \xi}}{\partial t}\right|^{2}+\left|\frac{\partial V_{\epsilon, \xi}}{\partial t}\right|^{2} d x \\
\leq & C(\phi)\left[\left\|\mu_{\epsilon, \xi}(0)\right\|+K_{\epsilon, \xi}^{\prime}(t)\right],
\end{aligned}
$$

for $\phi \in C_{0}^{1}\left(\mathbb{R}^{2}\right)$, where

$$
K_{\epsilon, \xi}(t)=\lambda_{\epsilon, \xi}^{-1} \int_{0}^{t} \int_{\Omega}\left|\frac{\partial U_{\epsilon, \xi}}{\partial t}\right|^{2}+\left|\frac{\partial V_{\epsilon, \xi}}{\partial t}\right|^{2} d x d t
$$

$C(\phi)$ is a positive constant depending only on $\|\phi\|_{C^{1}}^{2}$, and $\left\|\mu_{\epsilon, \xi}(0)\right\|$ denotes the total measures of $\mu_{\epsilon, \xi}(0)$.

By (4.1)-(4.3), it is easy to check that

$$
\frac{d}{d t} E_{\epsilon, \xi}\left(U_{\epsilon, \xi}, V_{\epsilon, \xi}\right)=-\lambda_{\epsilon, \xi}^{-1} \int_{\Omega}\left|\frac{\partial U_{\epsilon, \xi}}{\partial t}\right|^{2}+\left|\frac{\partial V_{\epsilon, \xi}}{\partial t}\right|^{2} .
$$

Hence by the assumption $\left(A_{4}\right)$, we obtain

$$
\lambda_{\epsilon, \xi}^{-1} \int_{0}^{T_{\epsilon, \xi}} \int_{\Omega}\left|\frac{\partial U_{\epsilon, \xi}}{\partial t}\right|^{2}+\left|\frac{\partial V_{\epsilon, \xi}}{\partial t}\right|^{2} d x d t \leq C(\xi, \Omega) .
$$

Moreover, by the assumptions $\left(A_{5}\right)$ and $\left(A_{6}\right)$, and Theorem II, we have

$$
\lambda_{\epsilon, \xi}^{-1} \int_{0}^{T_{\epsilon, \xi}} \int_{\Omega}\left|\frac{\partial U_{\epsilon, \xi}}{\partial t}\right|^{2}+\left|\frac{\partial V_{\epsilon, \xi}}{\partial t}\right|^{2} d x d t \leq \sqrt{\xi} C(\Omega)
$$

i.e.

$$
\left(\log \frac{1}{\epsilon}\right)^{-1} \int_{0}^{T_{\epsilon, \xi}} \int_{\Omega}\left|\frac{\partial U_{\epsilon, \xi}}{\partial t}\right|^{2}+\left|\frac{\partial V_{\epsilon, \xi}}{\partial t}\right|^{2} d x d t \leq C(\Omega) .
$$

Here we have used the assumption that $\lambda_{\epsilon, \xi}=\left(\log \frac{1}{\epsilon}\right) / \sqrt{\xi}$. Thus the function $E_{\epsilon, \xi}(\phi, t)-C(\phi)\left[\left\|\mu_{\epsilon, \xi}(0)\right\| \cdot t+K_{\epsilon, \xi}(t)\right]$ is monotonically nonincreasing for $t \in[0, \infty)$, where $E_{\epsilon, \xi}(\phi, t)=\int_{\Omega} \phi^{2} \mu_{\epsilon, \xi}(t) d x$. We choose a countable dense subset $\left\{\phi_{j}\right\}_{j=1}^{\infty}$ of $C_{0}^{1}\left(\mathbb{R}^{2}\right)$, and for each $j$ we can find a sequence of $\epsilon$ 's that goes to zero such that the corresponding sequence of functions $\eta_{\epsilon, \xi}^{j} \equiv E_{\epsilon, \xi}\left(\phi_{j}, t\right)-C\left(\phi_{j}\right)\left[\left\|\mu_{\epsilon, \xi}(0)\right\| \cdot t+K_{\epsilon, \xi}(t)\right]$ pointwise converges to a monotonically nonincreasing function $\eta_{\xi}^{j}(t)$ for each $t \geq 0$. Now we use the diagonal sequence to obtain a sequence of $\epsilon_{n} \downarrow 0$ such that $\eta_{\epsilon_{n}, \xi}^{j}(t)$ pointwise converges to a function $\eta_{\xi}(t)$ for each $t \geq 0$. It is then easy to see that $\left\|\mu_{\epsilon, \xi}(0)\right\| \rightarrow 2$ as $\epsilon \rightarrow 0+$. 
Step 2. The Radon measure obtained in step 1 is of the form $\mu(t)=\pi\left(\delta_{a_{\xi}(t)}+\delta_{b_{\xi}(t)}\right)$ for two distinct points $a_{\xi}(t)$ and $b_{\xi}(t)$ in $\Omega$. Then by the argument of step 2 and 3 in the proof of Theorem 2.1 (iii) in [12], we may obtain (4.13)-(4.15), and $a_{\xi}(t)$ and $b_{\xi}(t)$ are continuously dependent on $t \in[0, \infty)$ for $\xi>0$. Now we want to show that $a_{\xi}(t)$ and $b_{\xi}(t)$ converge uniformly on a finite time interval $t \in[0, T]$ as $\xi \rightarrow \infty$ (up to a subsequence), where $T>0$ is a positive constant. As for the step 3 of pp. 408 in [12], one may calculate

$$
\begin{aligned}
& \left|\frac{d}{d t} E_{\epsilon, \xi}(\phi, t)\right| \\
& \leq C(\phi) \lambda_{\epsilon, \xi}^{-2} \int_{\Omega}\left(\left|\frac{\partial U_{\epsilon, \xi}}{\partial t}\right|^{2}+\left|\frac{\partial V_{\epsilon, \xi}}{\partial t}\right|^{2}\right) d x \\
& \quad+C(\phi)\left[\lambda_{\epsilon, \xi}^{-1} \int_{\Omega}\left|\nabla U_{\epsilon, \xi}\right|^{2} d x\right]^{\frac{1}{2}}\left[\lambda_{\epsilon, \xi}^{-1} \int_{\Omega}\left|\frac{\partial U_{\epsilon, \xi}}{\partial t}\right|^{2} d x\right]^{\frac{1}{2}} \\
& \quad+C(\phi)\left[\lambda_{\epsilon, \xi}^{-1} \int_{\Omega}\left|\nabla V_{\epsilon, \xi}\right|^{2} d x\right]^{\frac{1}{2}}\left[\lambda_{\epsilon, \xi}^{-1} \int_{\Omega}\left|\frac{\partial V_{\epsilon, \xi}}{\partial t}\right|^{2} d x\right]^{\frac{1}{2}} \\
& \leq C(\phi) \lambda_{\epsilon, \xi}^{-2} \int_{\Omega}\left(\left|\frac{\partial U_{\epsilon, \xi}}{\partial t}\right|^{2}+\left|\frac{\partial V_{\epsilon, \xi}}{\partial t}\right|^{2}\right) d x \\
& \quad+C(\phi) \sqrt{\xi}\left[\left(\log \frac{1}{\epsilon}\right)^{-1} \int_{\Omega}\left|\frac{\partial U_{\epsilon, \xi}}{\partial t}\right|^{2}+\left|\frac{\partial V_{\epsilon, \xi}}{\partial t}\right|^{2} d x\right]^{\frac{1}{2}} .
\end{aligned}
$$

Hence by (4.19), we may obtain

$$
\int_{t_{1}}^{t_{2}}\left[\left(\log \frac{1}{\epsilon}\right)^{-1} \int_{\Omega}\left|\frac{\partial U_{\epsilon, \xi}}{\partial t}\right|^{2}+\left|\frac{\partial V_{\epsilon, \xi}}{\partial t}\right|^{2} d x\right]^{\frac{1}{2}} \leq\left|t_{1}-t_{2}\right|^{\frac{1}{2}} \sqrt{C(\Omega)} .
$$

By various proper choices of $\phi \in C_{0}^{1}(\Omega)$, as $\epsilon \rightarrow 0+($ up to a subsequence),

$$
\begin{aligned}
\left|a_{\xi}\left(t_{1}\right)-a_{\xi}\left(t_{2}\right)\right| & \leq\left|t_{1}-t_{2}\right|^{\frac{1}{2}} \sqrt{C(\Omega)}, \\
\left|b_{\xi}\left(t_{1}\right)-b_{\xi}\left(t_{2}\right)\right| & \leq\left|t_{1}-t_{2}\right|^{\frac{1}{2}} \sqrt{C(\Omega)},
\end{aligned}
$$

for $\xi>0$, where the constant $C(\Omega)$ may be different from that in (4.19). We may use the same notation for convenience. Therefore by Arzela-Ascoli Theorem, we may deduce the uniform convergence of $a_{\xi}$ and $b_{\xi}$ as $\xi \rightarrow \infty$ (up to a subsequence).

Step 3. Now we want to derive the motion equations of $a_{\xi}(t)$ and $b_{\xi}(t)$ as $\xi \rightarrow+\infty$. Without loss of generality, we may assume that $a=\left(a^{x}, 0\right), b=\left(b^{x}, 0\right), a_{\xi}(0)=a$, and $b_{\xi}(0)=b$ are located in the $x$-axis, where $a^{x}<b^{x}$. It suffices to show proof of (4.16) at $t=0$. For $0 \leq t \leq \delta^{*}$, we may assume

$$
\begin{aligned}
& a_{\xi}(t) \subset B_{\delta^{*}}(a), \\
& b_{\xi}(t) \subset B_{\delta^{*}}(b),
\end{aligned}
$$

where $\delta^{*}>0$ is a sufficiently small constant(see pp. 408 in [12]). For notation convenience, we may denote $a_{\xi}(t)$ and $b_{\xi}(t)$ as $a(t)$ and $b(t)$, respectively. For $R \in$ $\left[R_{0} / 2, R_{0}\right], R_{0}=\min \left\{\delta^{*} / 4,\left|a^{x}-b^{x}\right| / 16\right\}$, we multiply (4.1) by $\nabla U_{\epsilon, \xi},(4.2)$ by $\nabla V_{\epsilon, \xi}$, and integrate them respectively on $\Gamma_{R}^{ \pm}$, where $\Gamma_{R}^{ \pm}$are of $C^{1}$ domains satisfying

$$
\begin{aligned}
& \Gamma_{R}^{+} \subseteq B_{R}(b) \cup\left\{(x, y): \frac{1}{2}\left(a^{x}+b^{x}\right) \leq x \leq b^{x},-R \leq y \leq R\right\}, \\
& \Gamma_{R}^{-} \subseteq B_{R}(a) \cup\left\{(x, y): a^{x} \leq x \leq \frac{1}{2}\left(a^{x}+b^{x}\right),-R \leq y \leq R\right\},
\end{aligned}
$$


and

$$
\begin{aligned}
\partial \Gamma_{R}^{+} \supseteq\left\{(x, y) \in \partial B_{R}(b): x \geq b^{x}\right\} \\
\cup\left\{(x, y): x=\frac{1}{2}\left(a^{x}+b^{x}\right),-3 R / 4 \leq y \leq 3 R / 4\right\} \\
\cup\left\{(x, y): \frac{1}{2}\left(a^{x}+b^{x}\right)+\frac{1}{4} R \leq x \leq b^{x}, y= \pm R\right\}, \\
\partial \Gamma_{R}^{-} \supseteq\left\{(x, y) \in \partial B_{R}(a): x \leq a^{x}\right\} \\
\cup\left\{(x, y): x=\frac{1}{2}\left(a^{x}+b^{x}\right),-3 R / 4 \leq y \leq 3 R / 4\right\} \\
\cup\left\{(x, y): a^{x} \leq x \leq \frac{1}{2}\left(a^{x}+b^{x}\right)-\frac{1}{4} R, y= \pm R\right\} .
\end{aligned}
$$

Then we take sum of them and use integration by parts to obtain

$$
\begin{aligned}
& -\frac{\sqrt{\xi}}{\log \frac{1}{\epsilon}} \int_{\Gamma_{R}^{ \pm}} \frac{\partial U_{\epsilon, \xi}}{\partial t} \cdot \nabla U_{\epsilon, \xi}+\frac{\partial V_{\epsilon, \xi}}{\partial t} \cdot \nabla V_{\epsilon, \xi} d x \\
& =\frac{1}{4 \epsilon^{2}} \int_{\partial \Gamma_{R}^{ \pm}}\left[\left(1-\left|U_{\epsilon, \xi}\right|^{2}\right)^{2}+\left(1-\left|V_{\epsilon, \xi}\right|^{2}\right)^{2}\right] \nu \\
& +\frac{1}{2} \int_{\partial \Gamma_{R}^{ \pm}}\left(\left|\nabla U_{\epsilon, \xi}\right|^{2}+\left|\nabla V_{\epsilon, \xi}\right|^{2}\right) \nu \\
& -\int_{\partial \Gamma_{R}^{ \pm}}\left(\frac{\partial U_{\epsilon, \xi}}{\partial \nu} \cdot \nabla U_{\epsilon, \xi}+\frac{\partial V_{\epsilon, \xi}}{\partial \nu} \cdot \nabla V_{\epsilon, \xi}\right)+\frac{1}{2} \xi \int_{\partial \Gamma_{R}^{ \pm}}\left(U_{\epsilon, \xi} \cdot V_{\epsilon, \xi}\right)^{2} \nu .
\end{aligned}
$$

Here we have used the fact that $\lambda_{\epsilon, \xi}=-(\log \epsilon) / \sqrt{\xi}$.

On the other hand, we calculate with

$$
e_{\epsilon, \xi}(u, v)=\frac{1}{2}\left(|\nabla u|^{2}+|\nabla v|^{2}\right)+\frac{1}{4 \epsilon^{2}}\left[\left(1-|u|^{2}\right)^{2}+\left(1-|v|^{2}\right)^{2}\right]+\frac{1}{2} \xi(u \cdot v)^{2}
$$

that

$$
\begin{aligned}
\frac{\sqrt{\xi}}{\log \frac{1}{\epsilon}} \frac{d}{d t} \int_{\Gamma_{R}^{ \pm}} x e_{\epsilon, \xi}\left(U_{\epsilon, \xi}, V_{\epsilon, \xi}\right) d x \\
=-\frac{\xi}{\left(\log \frac{1}{\epsilon}\right)^{2}} \int_{\Gamma_{R}^{ \pm}} x\left(\left|\frac{\partial U_{\epsilon, \xi}}{\partial t}\right|^{2}+\left|\frac{\partial V_{\epsilon, \xi}}{\partial t}\right|^{2}\right) \\
-\frac{\sqrt{\xi}}{\log \frac{1}{\epsilon}} \int_{\Gamma_{R}^{ \pm}}\left(\frac{\partial U_{\epsilon, \xi}}{\partial t} \cdot \nabla U_{\epsilon, \xi}+\frac{\partial V_{\epsilon, \xi}}{\partial t} \cdot \nabla V_{\epsilon, \xi}\right) \\
+\frac{\sqrt{\xi}}{\log \frac{1}{\epsilon}} \int_{\partial \Gamma_{R}^{ \pm}} x\left(\frac{\partial U_{\epsilon, \xi}}{\partial \nu} \cdot \frac{\partial U_{\epsilon, \xi}}{\partial t}+\frac{\partial V_{\epsilon, \xi}}{\partial \nu} \cdot \frac{\partial V_{\epsilon, \xi}}{\partial t}\right) .
\end{aligned}
$$

Therefore, by integrating with respect to $R \in\left[R_{0} / 2, R_{0}\right]$ and with respect to t- 
variables, we get $f_{\epsilon, \xi}^{ \pm}(t)=g_{\epsilon, \xi}^{ \pm}(t)+h_{\epsilon, \xi}^{ \pm}(t)$, where

$$
\begin{aligned}
f_{\epsilon, \xi}^{ \pm}(t)= & \frac{\sqrt{\xi}}{\log \frac{1}{\epsilon}} \frac{2}{R_{0}} \int_{R_{0} / 2}^{R_{0}} \int_{\Gamma_{R}^{ \pm}} x \cdot\left[e_{\epsilon, \xi}\left(U_{\epsilon, \xi}, V_{\epsilon, \xi}\right)(t)-e_{\epsilon, \xi}\left(U_{\epsilon, \xi}, V_{\epsilon, \xi}\right)(0)\right] d x d R, \\
g_{\epsilon, \xi}^{ \pm}(t)= & \frac{2}{R_{0}} \int_{0}^{t} \int_{R_{0} / 2}^{R_{0}}\left(\int_{\partial \Gamma_{R}^{ \pm}} G_{\epsilon, \xi}\left(U_{\epsilon, \xi}, V_{\epsilon, \xi}\right)\right) d R d t, \\
G_{\epsilon, \xi}(u, v)= & \frac{1}{4 \epsilon^{2}}\left[\left(1-|u|^{2}\right)^{2}+\left(1-|v|^{2}\right)^{2}\right] \nu \\
& +\frac{1}{2}\left(|\nabla u|^{2}+|\nabla v|^{2}\right) \nu \\
& -\left(\frac{\partial u}{\partial \nu} \cdot \nabla u+\frac{\partial v}{\partial \nu} \cdot \nabla v\right)+\frac{1}{2} \xi(u \cdot v)^{2} \nu, \\
h_{\epsilon, \xi}^{ \pm}(t)= & -\frac{2}{R_{0}} \int_{R_{0} / 2}^{R_{0}} \int_{0}^{t} \frac{\xi}{\left(\log \frac{1}{\epsilon}\right)^{2}} \int_{\Gamma_{R}^{ \pm}} x\left(\left|\frac{\partial U_{\epsilon, \xi}}{\partial t}\right|^{2}+\left|\frac{\partial V_{\epsilon, \xi}}{\partial t}\right|^{2}\right) d x d t d R \\
& +\frac{2}{R_{0}} \int_{R_{0} / 2}^{R_{0}} \int_{0}^{t} \frac{\sqrt{\xi}}{\log \frac{1}{\epsilon}} \int_{\partial \Gamma_{R}^{ \pm}} x\left(\frac{\partial U_{\epsilon, \xi}}{\partial \nu} \cdot \frac{\partial U_{\epsilon, \xi}}{\partial t}+\frac{\partial V_{\epsilon, \xi}}{\partial \nu} \cdot \frac{\partial V_{\epsilon, \xi}}{\partial t}\right) d x d t d R .
\end{aligned}
$$

By (4.13) and (4.14), we may assume

$$
U_{\epsilon, \xi}(x, t)=u_{\epsilon, \xi}(x, t) e^{\frac{1}{2} i H_{\xi}(x)} \text { and } V_{\epsilon, \xi}(x, t)=v_{\epsilon, \xi}(x, t) e^{-\frac{1}{2} i H_{\xi}(x)} .
$$

Here $u_{\epsilon, \xi}$ and $v_{\epsilon, \xi}$ satisfy

$$
\begin{aligned}
& u_{\epsilon, \xi}(x, t) \rightarrow \frac{x-a_{\xi}(t)}{\left|x-a_{\xi}(t)\right|} e^{\frac{1}{2} i h_{\xi}(x)} \quad \text { weakly in } H_{l o c}^{1}\left(\bar{\Omega} \backslash\left\{a_{\xi}(t)\right\}\right), \\
& v_{\epsilon, \xi}(x, t) \rightarrow \frac{x-b_{\xi}(t)}{\left|x-b_{\xi}(t)\right|} e^{\frac{1}{2} i h_{\xi}(x)} \quad \text { weakly in } H_{l o c}^{1}\left(\bar{\Omega} \backslash\left\{b_{\xi}(t)\right\}\right),
\end{aligned}
$$

for each $\xi>0$ and $0 \leq t \leq T$ as $\epsilon \rightarrow 0+$, where $H_{\xi}(x)=\phi_{\xi}(x)-\Phi_{\xi}(x)$ and $h_{\xi}(x)=\phi_{\xi}(x)+\Phi_{\xi}(x)$. Hence we may obtain

$$
G_{\epsilon, \xi}\left(U_{\epsilon, \xi}, V_{\epsilon, \xi}\right)=G_{\epsilon, \xi}^{1}+G_{\epsilon, \xi}^{2}+G_{\epsilon, \xi}^{3},
$$

where

$$
\begin{aligned}
G_{\epsilon, \xi}^{1}= & \frac{1}{4 \epsilon^{2}}\left[\left(1-\left|u_{\epsilon, \xi}\right|^{2}\right)^{2}+\left(1-\left|v_{\epsilon, \xi}\right|^{2}\right)^{2}\right] \nu+\frac{1}{2}\left(\left|\nabla u_{\epsilon, \xi}\right|^{2}+\left|\nabla v_{\epsilon, \xi}\right|^{2}\right) \nu \\
& -\frac{\partial u_{\epsilon, \xi}}{\partial \nu} \cdot \nabla u_{\epsilon, \xi}-\frac{\partial v_{\epsilon, \xi}}{\partial \nu} \cdot \nabla v_{\epsilon, \xi}, \\
G_{\epsilon, \xi}^{2}= & \frac{1}{2}\left[\left(\nabla u_{\epsilon, \xi} \cdot i u_{\epsilon, \xi} \nabla H_{\xi}\right)-\left(\nabla v_{\epsilon, \xi} \cdot i v_{\epsilon, \xi} \nabla H_{\xi}\right)\right] \nu \\
& -\frac{1}{4}\left(\left|u_{\epsilon, \xi}\right|^{2}+\left|v_{\epsilon, \xi}\right|^{2}\right) \frac{\partial H_{\xi}}{\partial \nu} \nabla H_{\xi} \\
& -\frac{1}{2}\left(i u_{\epsilon, \xi} \frac{\partial H_{\xi}}{\partial \nu} \cdot \nabla u_{\epsilon, \xi}+\frac{\partial u_{\epsilon, \xi}}{\partial \nu} \cdot i u_{\epsilon, \xi} \nabla H_{\xi}\right) \\
& +\frac{1}{2}\left(i v_{\epsilon, \xi} \frac{\partial H_{\xi}}{\partial \nu} \cdot \nabla v_{\epsilon, \xi}+\frac{\partial v_{\epsilon, \xi}}{\partial \nu} \cdot i v_{\epsilon, \xi} \nabla H_{\xi}\right) \\
G_{\epsilon, \xi}^{3}= & {\left[\frac{1}{8}\left(\left|u_{\epsilon, \xi}\right|^{2}+\left|v_{\epsilon, \xi}\right|^{2}\right)\left|\nabla H_{\xi}\right|^{2}+\frac{1}{2} \xi\left(U_{\epsilon, \xi} \cdot V_{\epsilon, \xi}\right)^{2}\right] \nu . }
\end{aligned}
$$


By the same argument as pp. 409 in [12], we have

$$
\int_{R_{0} / 2}^{R_{0}} \int_{\partial \Gamma_{R}^{ \pm}}\left|G_{\epsilon, \xi}^{1}\right| \leq K_{0}
$$

and

$$
h_{\epsilon, \xi}^{ \pm}(t)=o_{\epsilon}(1)
$$

where $K_{0}$ is a positive constant independent of $\xi$. Here we have used the fact that $h_{\xi}(x)$ is a harmonic function satisfying the Neumann boundary condition $\frac{\partial h_{\xi}}{\partial \nu}=$ $-\frac{\partial}{\partial \nu}\left(\Theta_{a(t)}+\Theta_{b(t)}\right)$ on the boundary $\partial \Omega$.

Now we want to estimate the integral of $G_{\epsilon, \xi}^{3}$ on $\partial \Gamma_{R}^{ \pm}$. By (4.13) and (4.14), as $\epsilon \rightarrow 0+$, we may write the integral of $G_{\epsilon, \xi}^{3}$ as

$$
\int_{R_{0} / 2}^{R_{0}} \int_{\partial \Gamma_{R}^{ \pm}} G_{\epsilon, \xi}^{3}=\int_{R_{0} / 2}^{R_{0}} \int_{\partial \Gamma_{R}^{ \pm}}\left[\frac{1}{4}\left|\nabla H_{\xi}\right|^{2}+\frac{1}{2} \xi \cos ^{2}\left(\Theta_{a(t)}-\Theta_{b(t)}+H_{\xi}\right)\right] \nu+o_{\epsilon}(1)
$$

By the assumptions $\left(A_{5}\right)$ and $\left(A_{5}\right)$, Theorem II, and the energy dissipative formula (4.17), we have

$$
H_{\xi}+\Theta_{a(t)}-\Theta_{b(t)} \rightarrow \pi / 2 \text { exponentially in } \Omega \backslash L(t) \text { as } \xi \rightarrow \infty,
$$

and

$$
P_{\xi}\left(H_{\xi} ; a(t), b(t)\right)=c_{0}|L(t)|+o_{\xi}(1) \quad \text { as } \xi \rightarrow+\infty
$$

where $P_{\xi}$ is defined in $(3.7), L(t)$ is the line segment joining $a(t)$ and $b(t),|L(t)|$ is the length of $L(t)$, and $c_{0}$ is the positive constant defined in (3.11). Actually, by (4.15), when $\xi \rightarrow \infty$, the profile of $H_{\xi}$ crossing the domain wall $L(t)$ is preserved as time varies. From (4.20) and (4.30), we obtain

$$
\begin{aligned}
& \int_{\partial \Gamma_{R}^{+}} \cos ^{2}\left(\Theta_{a(t)}-\Theta_{b(t)}+H_{\xi}\right) \nu \\
& =\int_{\partial \Gamma_{R}^{+} \backslash S_{R}} \cos ^{2}\left(\Theta_{a(t)}-\Theta_{b(t)}+H_{\xi}\right) \nu \\
& +\int_{S_{R}} \cos ^{2}\left(\Theta_{a(t)}-\Theta_{b(t)}+H_{\xi}\right) \nu \\
& =\int_{S_{R}} \cos ^{2}\left(\Theta_{a(t)}-\Theta_{b(t)}+H_{\xi}\right) \nu+o_{\xi}(1),
\end{aligned}
$$

where

$$
S_{R}=\left\{(x, y): x=\frac{1}{2}\left(a^{x}+b^{x}\right),|y| \leq R / 8\right\}
$$

Besides,

$$
\int_{\partial \Gamma_{R}^{+}}\left|\nabla H_{\xi}\right|^{2} \nu=\int_{S_{R}}\left|\nabla H_{\xi}\right|^{2} \nu+O(1) \text { as } \xi \rightarrow \infty
$$


Hence

$$
\begin{aligned}
& \int_{\partial \Gamma_{R}^{+}}\left[\left|\nabla H_{\xi}\right|^{2}+\frac{1}{2} \xi \cos ^{2}\left(\Theta_{a(t)}-\Theta_{b(t)}+H_{\xi}\right)\right] \nu \\
& =\int_{S_{R}}\left\{\left|\nabla H_{\xi}\right|^{2}+\frac{1}{2} \xi \cos ^{2}\left(\Theta_{a(t)}-\Theta_{b(t)}+H_{\xi}\right)\right\}(-1,0) d y+O(1) .
\end{aligned}
$$

Similarly, by (4.21) and (4.30),

$$
\begin{aligned}
& \int_{\partial \Gamma_{R}^{-}}\left[\left|\nabla H_{\xi}\right|^{2}+\frac{1}{2} \xi \cos ^{2}\left(\Theta_{a(t)}-\Theta_{b(t)}+H_{\xi}\right)\right] \nu \\
& =\int_{S_{R}}\left\{\left|\nabla H_{\xi}\right|^{2}+\frac{1}{2} \xi \cos ^{2}\left(\Theta_{a(t)}-\Theta_{b(t)}+H_{\xi}\right)\right\}(1,0) d y+O(1) .
\end{aligned}
$$

Thus by (4.29)-(4.33), we obtain

$$
\frac{2}{R_{0}} \int_{R_{0} / 2}^{R_{0}} \int_{\partial \Gamma_{R}^{ \pm}} G_{\epsilon, \xi}^{3}= \pm \sqrt{\xi}\left(c_{0}^{\prime}+o_{\xi}(1)\right)(-1,0)+o_{\epsilon}(1)
$$

for $0 \leq t \leq \delta^{*}$, where $c_{0}^{\prime}$ is a positive constant depending only on the constant $c_{0}$ defined in (3.17).

By (4.24), (4.25) and (4.30), we have

$$
\frac{2}{R_{0}} \int_{R_{0} / 2}^{R_{0}} \int_{\partial \Gamma_{R}^{ \pm}}\left|G_{\epsilon, \xi}^{2}\right| \leq K_{1}
$$

where $K_{1}$ is a positive constant independent of $\epsilon$ and $\xi$. By step $2, f_{\epsilon_{n}, \xi}^{+}(t) \rightarrow$ $\pi \sqrt{\xi}(b(t)-b)$ and $f_{\epsilon_{n}, \xi}^{-}(t) \rightarrow \pi \sqrt{\xi}(a(t)-a)$ as $n \rightarrow \infty$. Hence by $(4.28), g_{\epsilon_{n}, \xi}^{+}(t) \rightarrow$ $\pi \sqrt{\xi}(b(t)-b)$ and $g_{\epsilon_{n}, \xi}^{-}(t) \rightarrow \pi \sqrt{\xi}(a(t)-a)$ as $n \rightarrow \infty$. Therefore by (4.27), (4.34) and (4.35), we may obtain (4.16) and complete the proof of Theorem IV.

Final Remark 1. In the time scale of order $O\left(\log \frac{1}{\epsilon}\right)$, vortices have already recombined in pairs to start with. So the motion of vortices is governed by the O.D.E. system which can be found in [11]. Since it is possible to locate the beginning positions of these paired vortices (they are basically various midpoints of the very initial locations of fractional degree vortices), one may easily create a situation in which these paired vortices motions are nontrivial as well.

Final Remark 2. In the time scale of order $O\left(\left(\log \frac{1}{\epsilon}\right) / \sqrt{\xi}\right)$, one may take double limits $\log \frac{1}{\epsilon} \gg \xi \rightarrow \infty$, and one may show the conclusions of Theorem IV remain true by the similar arguments.

Acknowledgement. The research of the first author is partially supported by the NSF grant DMS-0201443.The research of the second author is partially supported by a research Grant (NSC92-2115-M-194-014) from NSC of Taiwan.A big portion of the work was carried out while both authors were visiting the Center of the Mathematical Sciences at the Zhejiang University,Hangzhou,China.They wish to thank the center for the warm hospitality. 


\section{REFERENCES}

[1] N.D. Alikakos and G. Fusco, Slow dynamics for the Cahn-Hilliard equation in higher space dimensions: the motion of bubbles, Arch. Rational Mech. Anal., 141:1-61, 1998.

[2] E. Babaev, L.D. Faddeev, and A.J. Niemi, Hidden symmetry and knot solitons in a charged two-condensate Bose system, Phys. Rev. B, 65:100512-(1-4).

[3] F. Bethuel, H. Brezis, and F. Helein, Ginzburg-Landau Vortices, Birkhauser, 1994.

[4] E. Dumont and A.C. Mota, Unconventional vortex dynamics in superconducting states with broken time-reversal symmetry, Phys. Rev. B, 65:144519-(1-12).

[5] R. Fisher, S. Kim, B. Woodfield, N. Philips, N. Taillefer, K. Hasselback, J. Floquet, A. Giorgi, and J. Smith, Specific heat of $U_{P} t_{3}$ : Evidence for unconventional superconductivity, Phys. Rev. Lett., 62:1411-1414, 1989.

[6] V.B. Geshkenbein and A.I. Larkin, JETP Lett., 43:395, 1986.

[7] P.G. de Gennes, Superconductivity of metals and alloys, Addison-Wesley Pub. Com. INC, 1989.

[8] T. Ilmanen, Convergence of the Allen-Cahn equation to Brakke's motion by mean curvature, J. Diff. Geom., 38:417-461, 1993.

[9] J.R. Kirtley, M.B. Ketchen, K.G. Stawiaz, J.Z. Sun, W.J. Gallagher, S.H. Blanton, and S.J. Wind, Appl. Phys. Lett., 66:1138, 1995.

[10] F.H. Lin, Some dynamic properties of Ginzburg-Landau vortices, Comm. Pure Appl. Math., 49:323-359, 1996, and A remark on the previous paper: Some dynamical properties of Ginzburg-Landau vortices, Comm. Pure Appl. Math., 49:361-364, 1996.

[11] F.H. Lin, Static and moving vortices in Ginzburg-Landau theories, progress in nonlinear differential equations and their applications, vol. 29, 1997, Birkhäuser Verlag Basel/ Switzerland.

[12] F.H. Lin, Complex Ginzburg-Landau equations and dynamics of vortices, filaments, and codimension-2 submanifolds, Comm. Pure Appl. Math., 51(4):385-441, 1998.

[13] F.H. Lin, Vortex dynamics for the nonlinear wave equation, Comm. Pure Appl. Math., LII(6):737-761, 1999.

[14] F.H. Lin and J.X. Xin, On the incompressible fluid limit and the vortex motion law of the nonlinear Schrödinger equation, Comm. Math. Phys., 200(2):249-274, 1999.

[15] L. Modica, The gradient theory of phase transitions and the minimal interface criterion, Arch. Rational Mech. Anal., 98(2):123-142, 1987.

[16] L. Pitaevskii and S. Stringari, The Quest for superfluidity in Fermi gases, Science, 298:21442146,2002

[17] M. Sigrist and D.F. Agterberg, The role of domain walls on the vortex creep dynamics in unconventional superconductors, Prog. Theor. Phys., 102:965, 1999.

[18] M. Sigrist, T.M. Rice, and K. Ueda, Low-field magnetic response of complex superconductors, Phys. Rev. Lett., 63:1727-1730, 1989.

[19] M. Sigrist and K. Ueda, Phenomenological theory of conventional superconductivity, Rev. Mod. Phys., 63(2):239-311, 1991.

[20] D.T. Son and M.A. Stephanov, Domain walls of relative phase in two-component BoseEinstein condensates, Phys. Rev. A, 65: 063621-(1-10), 2002.

[21] P. Sternberg, The effect of a singular perturbation on nonconvex variational problems, Arch. Rational Mech. Anal., 101(3):209-260, 1988.

[22] J.X. Zhu, C.S. Ting, J.L. Shen, and Z.D. Wang, Ginzburg-Landau equations for layered p-wave superconductors, Phys. Rev. B, 56(21):14093-14101, 1997. 